\title{
COMBINATORICS AND GEOMETRY OF TRANSPORTATION POLYTOPES: AN UPDATE
}

\author{
JESÚS A. DE LOERA AND EDWARD D. KIM
}

\begin{abstract}
A transportation polytope consists of all multidimensional arrays or tables of non-negative real numbers that satisfy certain sum conditions on subsets of the entries. They arise naturally in optimization and statistics, and also have interest for discrete mathematics because permutation matrices, latin squares, and magic squares appear naturally as lattice points of these polytopes.

In this paper we survey advances on the understanding of the combinatorics and geometry of these polyhedra and include some recent unpublished results on the diameter of graphs of these polytopes. In particular, this is a thirty-year update on the status of a list of open questions last visited in the 1984 book by Yemelichev, Kovalev and Kravtsov and the 1986 survey paper of Vlach.
\end{abstract}

\section{INTRODUCTION}

Transportation polytopes are well-known objects in mathematical programming and statistics. In the operations research literature, classical transportation problems arise from the problem of transporting goods from a set of factories, each with given supply outcome, and a set of consumer centers, each with an amount of demand. Assuming the total supply equals the total demand and that costs are specified for each possible pair (factory, consumer center), one may wish to optimize the cost of transporting goods. Indeed this was the original motivation that led Kantorovich (see [100]), Hitchcock (see [94]), and T. C. Koopmans (see [106]) to look at these problems. They are indeed among the first linear programming problems investigated, and Koopmans received the Nobel Prize in Economics for his work in this area (see 95] for an interesting historical perspective). Not much later Birkhoff (see [17]), von Neumann (see [144]), and Motzkin (see, e.g., [117]) were key contributors to the topic. The success of combinatorial algorithms such as the Hungarian method (see [7, 21, 72, 73, 102, 107, 108, 118, 138]) depends on the rich combinatorial structure of the convex polyhedra that defined the possible solutions, the so called transportation polytopes.

In statistics, people have looked at the integral transportation tables, which are widely known as contingency tables. In statistics, a contingency table represents sample data arranged or tabulated by categories of combined properties. Several questions motivate the study of the geometry of contingency tables, for instance, in the table entry security problem: given a table $T$ (multi-dimensional perhaps) with statistics on private data about individuals, we may wish to release aggregated marginals of such a table without disclosing information about the exact entries of the table. What can a data thief discover about $T$ from the published marginals? When is $T$ uniquely identifiable by its margins? This problem has been studied by

2010 Mathematics Subject Classification. 37F20, 52B05, 90B06, 90C08. 
many researchers (see $34,42,46,47,66,67,68,74,98$ and the references therein). Another natural problem is whether a given table presents strong evidence of significant relations between the characteristics tabulated (e.g., is cancer related to smoking). There is a lot of interest among statisticians on testing significance of independence for variables. Some methods depend on counting all possible contingency tables with given margins (see e.g., 65, 113]). This in turn is an interesting combinatorial geometric problem on the lattice points of transportation polytopes.

In this article we survey the state of the art in the combinatorics and geometry of transportation polytopes and contingency tables. The survey [141] by Vlach, the 1984 monograph [146 by Yemelichev, Kovalev, and Kravtsov, and the paper 103 by Klee and Witzgall summarized the status of transportation polytopes up to the 1980s. Due to recent advances on the topic by the authors and others, we decided to write a new updated survey collecting remaining open problems and presenting recent solutions. We also included details on some unpublished new work on the diameter of the graphs of these polytopes.

In what follows we will denote by $[q]=\{1,2, \ldots, q\}$. Similarly $\mathbb{R}_{\geq 0}^{n}$ denotes those vectors in $\mathbb{R}^{n}$ whose entries are non-negative. Our notation and terminology on polytopes follows [85] and [147].

\section{Classical transportation polytopes (2-Ways)}

We begin by introducing the most well-known subfamily, the classical transportation polytopes in just two indices. We call them 2-way transportation polytopes and in general $d$-ways refers to the case of variables with $d$ indices. Many of these facts are well-known and can be found in 146, but we repeat them here as we will use them in what follows.

Fix two integers $p, q \in \mathbb{Z}_{>0}$. The transportation polytope $P$ of size $p \times q$ defined by the vectors $u \in \mathbb{R}^{p}$ and $v \in \mathbb{R}^{q}$ is the convex polytope defined in the $p q$ variables $x_{i, j} \in \mathbb{R}_{\geq 0}(i \in[p], j \in[q])$ satisfying the $p+q$ equations

$$
\sum_{j=1}^{q} x_{i, j}=u_{i}(i \in[p]) \quad \text { and } \quad \sum_{i=1}^{p} x_{i, j}=v_{j}(j \in[q]) .
$$

Since the coordinates $x_{i, j}$ of $P$ are non-negative, the conditions (2.1) imply $P$ is bounded. The vectors $u$ and $v$ are called marginals or margins. These polytopes are called transportation polytopes because they model the transportation of goods from $p$ supply locations (with the $i$ th location supplying a quantity of $u_{i}$ ) to $q$ demand locations (with the $j$ th location demanding a quantity of $v_{j}$ ). The feasible points $x=\left(x_{i, j}\right)_{i \in[p], j \in[q]}$ in a $p \times q$ transportation polytope $P$ model the scenario where a quantity of $x_{i, j}$ of goods is transported from the $i$ th supply location to the $j$ th demand location. See Figure 1 .

Example 2.1. Let us consider the $3 \times 3$ transportation polytope $P_{3 \times 3}$ defined by the marginals $u=(5,5,1)^{T}$ and $v=(2,7,2)^{T}$, which corresponds to the transportation problem shown in Figure 1. A point $x^{*}=\left(x_{i, j}^{*}\right)$ in $P$ is shown in Figure 2, The equations in 2.1) are conditions on the row sums and column sums (respectively) of tables $x \in P$.

2.1. Dimension and feasibility. Notice in Example 2.1 that $5+5+1=2+7+2$. The condition that the sum of the supply margins equals the sum of the demand 
supplies by factories

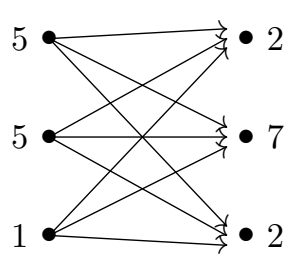

demands

on three cities

Figure 1. Three supplies and three demands

$$
x^{*}=\begin{array}{|l|l|l|}
\hline x_{1,1}^{*} & x_{1,2}^{*} & x_{1,3}^{*} \\
\hline x_{2,1}^{*} & x_{2,2}^{*} & x_{2,3}^{*} \\
\hline x_{3,1}^{*} & x_{3,2}^{*} & x_{3,3}^{*} \\
\hline
\end{array}=\begin{array}{|l|l|l|}
\hline 2 & 2 & 1 \\
\hline 0 & 5 & 0 \\
\hline 0 & 0 & 1 \\
\hline
\end{array}
$$

Figure 2. A point $x^{*} \in P_{3 \times 3}$

margins is not only necessary but also sufficient for a classical transportation polytope to be non-empty:

Lemma 2.2. Let $P$ be the $p \times q$ classical transportation polytope defined by the marginals $u \in \mathbb{R}_{\geq 0}^{p}$ and $v \in \mathbb{R}_{\geq 0}^{q}$. The polytope $P$ is non-empty if and only if

$$
\sum_{i \in[p]} u_{i}=\sum_{j \in[q]} v_{j}
$$

The proof of this lemma uses the well-known northwest corner rule algorithm (see [127] or Exercise 17 in Chapter 6 of [146]).

The equations 2.1 and the inequalities $x_{i, j} \geq 0$ can be rewritten in the matrix form

$$
P=\left\{x \in \mathbb{R}^{p q} \mid A x=b, x \geq 0\right\}
$$

with a $0-1$ matrix $A$ of size $(p+q) \times p q$ and a vector $b \in \mathbb{R}^{p+q}$ called the constraint matrix. The constraint matrix for a $p \times q$ transportation polytope is the vertex-edge incidence matrix of the complete bipartite graph $K_{p, q}$.

Lemma 2.3. Let $A$ be the constraint matrix of a $p \times q$ transportation polytope $P$. Then:

(1) Maximal rank submatrices of $A$ correspond to spanning trees on $K_{p, q}$.

(2) $\operatorname{rank}(A)=p+q-1$.

(3) Each subdeterminant of $A$ is \pm 1 , thus $A$ is totally unimodular.

(4) If $P \neq \emptyset$, its dimension is $p q-(p+q-1)=(p-1)(q-1)$.

Part 4 follows from Part 2 ,

Example 2.4. Continuing from Example 2.1 observe $P_{3 \times 3}=\left\{x \in \mathbb{R}^{9} \mid A_{3 \times 3} x=\right.$ $b, x \geq 0\}$, where $A_{3 \times 3}$ is the constraint matrix

$$
A_{3 \times 3}=\left[\begin{array}{ccccccccc}
1 & 0 & 0 & 1 & 0 & 0 & 1 & 0 & 0 \\
0 & 1 & 0 & 0 & 1 & 0 & 0 & 1 & 0 \\
0 & 0 & 1 & 0 & 0 & 1 & 0 & 0 & 1 \\
1 & 1 & 1 & 0 & 0 & 0 & 0 & 0 & 0 \\
0 & 0 & 0 & 1 & 1 & 1 & 0 & 0 & 0 \\
0 & 0 & 0 & 0 & 0 & 0 & 1 & 1 & 1
\end{array}\right] \quad \text { and } \quad b=\left[\begin{array}{c}
2 \\
7 \\
2 \\
5 \\
5 \\
1
\end{array}\right] .
$$


Up to permutation of rows and columns, the matrix $A_{3 \times 3}$ is the unique constraint matrix for $3 \times 3$ classical transportation polytopes. It is a $6 \times 9$ matrix of rank five. Thus, $P_{3 \times 3}$ is a four-dimensional polytope described in a nine-dimensional ambient space.

Birkhoff polytopes, first introduced by G. Birkhoff in [17, are an important subclass of transportation polytopes:

Definition 2.5. The pth Birkhoff polytope, denoted by $B_{p}$, is the $p \times p$ classical transportation polytope with margins $u=v=(1,1, \ldots, 1)^{T}$.

The Birkhoff polytope is also called the assignment polytope or the polytope of doubly stochastic matrices (see, e.g., [6]). It is the perfect matching polytope of the complete bipartite graph $K_{p, p}$. We can generalize the definition of the Birkhoff polytope to rectangular arrays:

Definition 2.6. The central transportation polytope is the $p \times q$ classical transportation polytope with $u_{1}=\cdots=u_{p}=q$ and $v_{1}=\cdots=v_{q}=p$. This polytope is also called the generalized Birkhoff polytope of size $p \times q$.

2.2. Combinatorics of faces and graphs. The study of the faces of transportation polytopes is a nice combinatorial question (see, e.g., 9]). Unfortunately it is still incomplete, e.g., one does not know the number of $i$-dimensional faces of each dimension other than in a few cases. E.g., in [123, Pak presented an efficient algorithm for computing the $f$-vector of the generalized Birkhoff polytope of size $p \times(p+1)$. Hartfiel (see [89]) and Dahl (see [53]) described the supports of certain feasible points in classical transportation polytopes. In this section, we fully describe the vertices and the edges of a 2 -way transportation polytope $P$. The resulting graph has some interesting properties, but there are still open questions about it.

Let $P$ be a $p \times q$ classical transportation polytope. For a point $x=\left(x_{i, j}\right)_{i \in[p], j \in[q]}$, define the support set $\operatorname{supp}(x)=\left\{(i, j) \in[p] \times[q] \mid x_{i, j}>0\right\}$. We also define a bipartite graph $B(x)$, called the support graph of $x$. The graph $B(x)$ is the following subgraph of the complete bipartite graph $K_{p, q}$ :

- Vertices of $B(x)$. The vertices of the graph $B(x)$ are the vertices of the complete bipartite graph $K_{p, q}$. We label the supply nodes $\sigma_{1}, \ldots, \sigma_{p}$ and the demand nodes $\delta_{1}, \ldots, \delta_{q}$.

- Edges of $B(x)$. There is an edge $\left(\sigma_{i}, \delta_{j}\right)$ if and only if $x_{i, j}$ is strictly positive. In other words, the edge set is indexed by $\operatorname{supp}(x)$.

Example 2.7. Let us consider the point $x^{*} \in P_{3 \times 3}$ from Example 2.1. Here, $\operatorname{supp}\left(x^{*}\right)=\left\{\left(\sigma_{1}, \delta_{1}\right),\left(\sigma_{1}, \delta_{2}\right),\left(\sigma_{1}, \delta_{3}\right),\left(\sigma_{2}, \delta_{2}\right),\left(\sigma_{3}, \delta_{3}\right)\right\}$. Figure 3 depicts the graph $B\left(x^{*}\right)$.

An important subclass of transportation polytopes are those which are generic. Generic transportation polytopes are easiest to analyze in the proofs which follow and are the ones typically appearing in applications. Generic $d$-way transportation polytopes are those whose vertices have maximal possible non-zero entries. All generic transportation polytopes are simple, but not vice versa.

Definition 2.8. A $p \times q$ classical transportation polytope $P$ is generic if

$$
\sum_{i \in Y} u_{i} \neq \sum_{j \in Z} v_{j}
$$




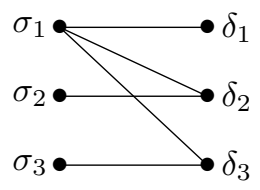

Figure 3 . The support graph $B\left(x^{*}\right)$ of the point $x^{*} \in P_{3 \times 3}$. The nodes of $B\left(x^{*}\right)$ on the left are the $p=3$ supplies. The nodes on the right are the $q=3$ demands.

for every non-empty proper subset $Y \subsetneq[p]$ and non-empty proper subset $Z \subsetneq[q]$. (Of course, due to $(2.2)$, we must disallow the case where $Y=[p]$ and $Z=[q]$.)

The graph properties of $B(x)$ provide a useful combinatorial characterization of the vertices of classical transportation polytopes:

Lemma 2.9 (Klee, Witzgall [103]). Let $P$ be a $p \times q$ classical transportation polytope defined by the marginals $u \in \mathbb{R}_{>0}^{p}$ and $v \in \mathbb{R}_{>0}^{q}$, and let $x \in P$. Then the graph $B(x)$ is spanning. The point $x$ is a vertex of $P$ if and only if $B(x)$ is a spanning forest. Moreover, if $P$ is generic, then $x$ is a vertex of $P$ if and only if $B(x)$ is a spanning tree.

Corollary 2.10. Let $x$ be a point in a generic $p \times q$ classical transportation polytope $P$. Then $x$ is a vertex of $P$ if and only if $|\operatorname{supp}(x)|=p+q-1$.

A vertex of a $p \times q$ transportation polytope is non-degenerate if it has $p+q-1$ positive entries. Otherwise, the vertex is degenerate. A transportation polytope is non-degenerate if all its vertices are non-degenerate. Non-degenerate transportation polytopes are of particular interest, as they have the largest possible number of vertices and largest possible diameter among the graphs of all transportation polytopes of given type and parameters (e.g., $p, q$, and $s$ ). Indeed, if $P$ is a degenerate transportation polytope, by carefully perturbing the marginals that define $P$ we can get a non-degenerate polytope $P^{\prime}$. (A careful explanation of how to do the perturbation is given in Lemma 4.6 of Chapter 6 in [146] on page 281.) The perturbed marginals are obtained by taking a feasible point $x$ in $P$, perturbing the entries in the table and using the recomputed sums as the new marginals for $P^{\prime}$. The graph of $P$ can be obtained from that of $P^{\prime}$ by contracting certain edges, which cannot increase either the diameter nor the number of vertices.

Finally, note the following property on the vertices of a classical transportation polytope, which follows from part 3 of Lemma 2.3 and Cramer's rule:

Corollary 2.11. Given integral marginals $u, v$, all vertices of the corresponding transportation polytope are integral.

We now recall a classical characterization of the vertices of the Birkhoff polytope:

Theorem 2.12 (Birkhoff-von Neumann Theorem). The $p$ ! vertices of the pth Birkhoff polytope $B_{p}$ are the 0-1 permutation matrices of size $p \times p$.

In other words, the vertices of the Birkhoff polytope are the permutation matrices, so every doubly stochastic matrix is a convex combination of permutation matrices. This theorem was proved by Birkhoff in 17 and proved independently by 
von Neumann (see 144). Equivalent results were shown earlier in the thesis 136] of Steinitz, and the theorem also follows from [104] and [105] by König. For a more complete discussion, see the preface to [111. See also the papers [25, 26, 27, 28, where various various combinatorial and geometric properties of the Birkhoff polytope were studied such as its graph. Of course due to the above theorem, Birkhoff's polytopes play an important role in combinatorics and discrete optimization and the literature about their properties is rather large.

We also want to know how many vertices a transportation polytope can have. In particular there is a visible difference in behavior between generic and nongeneric polytopes. How about maximum number of vertices? The exact formula is complicated but the following result of Bolker in [18, can serve as a reference:

Lemma 2.13 (Bolker, [18). The maximum possible number of vertices among $p \times q$ transportation polytopes is achieved by the central transportation polytope whose marginals are $u=(q, q, \ldots, q)$ and $v=(p, p, \ldots, p)$.

Indeed one can characterize which transportation polytopes reach the largest possible number of vertices. (See results by Yemelichev, Kravtsov and collaborators from the 1970's mentioned in [146].)

Question 2.14. What are the possible values for the number of vertices of a generic $p \times q$ transportation polytope? Are there gaps or do all integer values on a interval occur?

A partial answer to this question is provided in Table 1 , with more detail available at [139. Another partial answer, given in [58, is:

Theorem 2.15. The number of vertices of a non-degenerate $p \times q$ classical transportation polytope is divisible by $\operatorname{gcd}(p, q)$.

\begin{tabular}{|c|c|}
\hline sizes & Distribution of number of vertices in transportation polytopes \\
\hline $2 \times 3$ & 3456 \\
\hline $2 \times 4$ & 4681012 \\
\hline $2 \times 5$ & 5811121415161718192021222324252627282930 \\
\hline $3 \times 3$ & 9121518 \\
\hline $3 \times 4$ & 162124262729313234363739404142444546484950 \\
& 52535456575860616263646667687071727475767880849096 \\
\hline $4 \times 4$ & 108116124128136140144148152156160164168172176180184188192 \\
& 196200204208212216220224228232236240244248252256260264268 \\
& 272276280284288296300304312320340360 \\
\hline
\end{tabular}

TABLE 1. Numbers of vertices of $p \times q$ transportation polytopes

The support graph associated to a point of the transportation polytope also characterizes edges of classical transportation polytopes. (See Lemma 4.1 in Chapter 6 of [146].)

Proposition 2.16. Let $x$ and $x^{\prime}$ be distinct vertices of a classical transportation polytope $P$. Then the vertices $x$ and $x^{\prime}$ are adjacent if and only if the graph $B(x) \cup$ $B\left(x^{\prime}\right)$ contains a unique cycle. 
This can be seen since the bases corresponding to the vertices $x$ and $x^{\prime}$ differ in the addition and the removal of one element (see [112, 130]).

One can also characterize the facets of the $p \times q$ transportation polytope, which have dimension $(p-1)(q-1)-1$ by Lemma 2.3. The following lemma is Theorem 3.1 in Chapter 6 of [146].

Lemma 2.17. Let $P$ be the $p \times q$ transportation polytope $(p q>4)$ defined by marginals $u$ and $v$. Pick integers $1 \leq i^{*} \leq p$ and $1 \leq j^{*} \leq q$. The subset of points of $P$

$$
F_{i^{*}, j^{*}}=\left\{\left(x_{i, j}\right) \in P \mid x_{i^{*}, j^{*}}=0\right\}
$$

is a facet of $P$ if and only if $u_{i^{*}}+v_{j^{*}}<\sum_{i=1}^{p} u_{i}$.

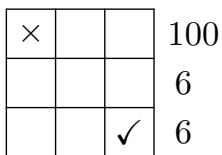

383737

Figure 4 . The equation $x_{3,3}=0$ defines a facet, while the top-left corner entry corresponds to an equation $x_{1,1}=0$ that does not.

See Figure 4 for an example. From this basic characterization we see:

Corollary 2.18. For $2 \leq p \leq q$ and $q \geq 3$, the possible number of facets of a $p \times q$ transportation polytope is a number of the form $(p-1) q+k$ for $k=0, \ldots, q$ and only such integers can occur.

For example, $3 \times 3$ transportation polytopes can have 6,7 , 8 , or 9 facets and only these values occur.

2.2.1. Diameter of graphs of transportation polytopes. Now we study a classical question about the graphs of transportation polytopes. Recall that the distance between two vertices $x, y$ of a polytope $P$ is the minimal number $\operatorname{dist}_{P}(x, y)$ of edges needed to go from $x$ to $y$ in the graph of $P$. The diameter of a polytope is the maximum possible distance between pairs of vertices in the graph of the polytope. Though the Hirsch Conjecture was finally shown to be false in general for polytopes (see [128]), the problem is still unsolved for transportation polytopes, and diameter bounds for this special class of polytopes are very interesting. Dyer and Frieze (see 69]) gave the first polynomial diameter bound for totally unimodular polytopes which applies to classical transportation polytopes (and more generally to network polytopes), but this was recently improved by Bonifas et al. in [19].

The diameters of classical transportation polytopes and their applications (see, e.g., 49]) have been studied extensively. In [8], Balinski proved that the Hirsch Conjecture holds and is tight for dual transportation polyhedra. For the specific case of transportation polytopes Yemelichev, Kovalev, and Kravtsov (see Theorem 4.6 in Chapter 6 of [146] and the references therein) and Stougie (see [137]) presented improved polynomial bounds. This was improved to a quadratic bound by van den Heuvel and Stougie in [140, and further improved to a linear bound: 
Theorem 2.19 (Brightwell, van den Heuvel, Stougie [22]). The diameter of every $p \times q$ transportation polytope is at most $8(p+q-2)$.

The bound follows from a crucial lemma which bounds the graph distance $\operatorname{dist}_{P}\left(y, y^{\prime}\right)$ between any two vertices $x$ and $y$ of a $p \times q$ transportation polytope $P$, by constructing vertices $x^{\prime}$ and $y^{\prime}$ of $P$ and nodes $\sigma, \delta$ of $K_{p, q}$ such that $\operatorname{deg}_{B\left(x^{\prime}\right)}(\delta)=$ $\operatorname{deg}_{B\left(y^{\prime}\right)}(\delta)=1,(\sigma, \delta) \in B\left(x^{\prime}\right) \cap B\left(y^{\prime}\right)$, and $\operatorname{dist}_{P}\left(x, x^{\prime}\right)+\operatorname{dist}_{P}\left(y, y^{\prime}\right) \leq 8$. In the arguments below, there is an important distinction between vertices of the polytope $P$ (which we always denote by $x$ or $y$ ) and nodes of the support graph $B(x) \subset K_{p, q}$ of a vertex $x$ of $P$ (which we always denote by $\sigma$ or $\delta$ ).

Theorem 2.19 was further improved by Cor Hurkens 97 .

Theorem 2.20 (Hurkens [97]). The diameter of every $p \times q$ transportation polytope is at most $4(p+q-2)$.

We present a brief sketch of Hurkens' proof. The result follows immediately from this lemma:

Lemma 2.21 (Hurkens 97]). For any two vertices $x$ and $y$ of $a p \times q$ transportation polytope $P$, there is an integer $r \geq 1$, a vertex $y^{\prime}$ of $P$, and nodes $\sigma, \delta_{1}, \ldots, \delta_{r}$ of $K_{p, q}$ such that:

(1) $\operatorname{deg}_{B(x)}\left(\delta_{k}\right)=\operatorname{deg}_{B\left(y^{\prime}\right)}\left(\delta_{k}\right)=1$ for $k=1, \ldots, r$,

(2) $\left(\sigma, \delta_{k}\right) \in B(x), B\left(y^{\prime}\right)$ for $k=1, \ldots, r$, and

(3) $\operatorname{dist}_{P}\left(y, y^{\prime}\right) \leq 4 r$.

The key idea that Hurkens showed is that four pivots are required (on average) to construct a common leaf node. More specifically, Hurkens proved this lemma by showing that for any two vertices $x$ and $y$ of a transportation polytope $P$, there is a node $\sigma$ in $K_{p, q}$ (which can be assumed to be a supply) with $r$ incident edges $\left(\sigma, \delta_{1}\right), \ldots,\left(\sigma, \delta_{r}\right)$ in $B(x)$ where $\delta_{1}, \ldots, \delta_{r}$ are all leaf nodes (which are necessarily demands) of $K_{p, q}$. Moreover, the nodes $\sigma, \delta_{1}, \ldots, \delta_{r}$ of $K_{p, q}$ identified in Hurkens' algorithm also satisfy the property that if

$$
S:=\left\{\left(\sigma, \delta_{k}\right) \mid\left(\sigma, \delta_{k}\right) \in B(y), k=1, \ldots, r\right\},
$$

then there is a vertex $y^{\prime}$ of $P$ obtained after at most $4 r$ pivots from the vertex $y$ of $P$ such that $B(x)$ and $B\left(y^{\prime}\right)$ have $r$ common leaf nodes.

In the algorithm of Brightwell, van den Heuvel, and Stougie (see 22]), pivots are applied to vertices $x$ and $y$ of $P$, resulting in new vertices $x^{\prime}$ and $y^{\prime}$ of $P$. A key difference in Hurkens' algorithm in [97. is that pivots are only applied to one of the two vertices $x$ and $y$ of $P$. Without loss of generality, pivots are applied to the vertex $y$ of $P$ and not applied to the vertex $x$ of $P$. Thus, we do not describe the vertex $x$ further. Other than the property that the demand nodes $\delta_{1}, \ldots, \delta_{r}$ are leaf nodes in $B(x)$ adjacent to the node $\sigma$, the structure of $B(x)$ may be arbitrary.

We label the relevant supply and demands nodes participating in pivots. For each $k=1, \ldots, r$ let $\left(\sigma_{k, n}, \delta_{k}\right)$ for $n=1, \ldots, \ell_{k}$ be the edges in $B(y) \backslash S$ incident to $\delta_{k}$, where $\ell_{k}=\operatorname{deg}_{B(y) \backslash S}\left(\delta_{k}\right)$. Let $\left(\sigma, c_{q}\right)$ be the edges in $B(y) \backslash S$ incident to $\sigma$ for $q=1, \ldots, t$ where $t=\operatorname{deg}_{B(y) \backslash S}(\sigma)$. See Figure 5 . Here we describe the successive pivots applied starting from the vertex $y$ of $P$. For each $k=1, \ldots, r$, we do the following: 


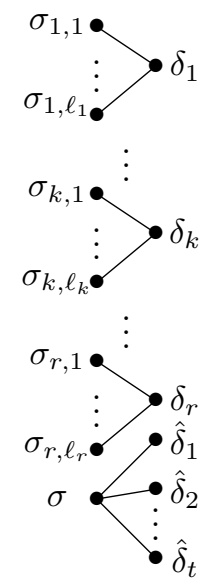

Figure 5. Example of supply nodes adjacent to demand nodes $\delta_{k}$ in $B(y)$ for $k=1, \ldots, r$ and demand nodes adjacent to the supply node $\sigma$

(1) If $\left(\sigma, \delta_{1}\right)$ is not in the support graph, pivot to add $\left(\sigma, \delta_{1}\right)$. Then, pivot to add edges of the form $\left(\sigma_{1, n}, \hat{\delta}_{q}\right)$ for $n=1,2, \ldots$ until all edges of the form $\left(\sigma_{1, n}, \delta_{1}\right)$ are removed.

(2) If $\left(\sigma, \delta_{2}\right)$ is not in the support graph, pivot to add $\left(\sigma, \delta_{2}\right)$. Then, pivot to add edges of the form $\left(\sigma_{2, n}, \hat{\delta}_{q}\right)$ for $n=1,2, \ldots$ until all edges of the form $\left(\sigma_{2, n}, \delta_{2}\right)$ are removed.

(3) Continue in this way for $k=3, \ldots, r$ : If $\left(\sigma, \delta_{k}\right)$ is not in the support graph, pivot to add it. Then, pivot to add edges of the form $\left(\sigma_{k, n}, \hat{\delta}_{q}\right)$ for $n=1,2, \ldots$ until all edges of the form $\left(\sigma_{k, n}, \delta_{k}\right)$ are removed.

In the resulting vertex $y^{\prime}$ of $P$, the support graph $B\left(y^{\prime}\right)$ has $\delta_{1}, \ldots, \delta_{r}$ as leaf nodes adjacent to $\sigma$, which matches the support graph $B(x)$ of the vertex $x$ of $P$. What remains to show (and we skip it) is that there is a choice of nodes $\sigma, \delta_{1}, \ldots, \delta_{r}$ where the number of pivots performed is at most $4 r$. Instead, we illustrate the idea behind the sequence of prescribed pivots in an example:

Example 2.22. Let $y$ be a vertex of $P$ where nodes $\sigma, \delta_{1}, \ldots, \delta_{r}$ in $B(y)$ are already identified. Figure 6 shows the support graph $B(y)$. (The vertex $x$ and its associated support graph $B(x)$ can be arbitrary, thus we do not depict it in Figure 6, )

Since $\left(\sigma, \delta_{1}\right)$ is not in the support graph $B(y)$ of the vertex $y$ of $P$, we insert it, and the pivot operation removes the edge $\left(\sigma_{1,3}, \delta_{1}\right)$. We now apply pivots to the resulting adjacent vertex of $P$ as follows: After the pivot, only the edges $\left(\sigma_{1,1}, \delta_{1}\right)$ and $\left(\sigma_{1,2}, \delta_{1}\right)$ are incident to the demand node $\delta_{1}$. These two edges are removed by pivoting to add the edges $\left(\sigma_{1,1}, \hat{\delta}_{1}\right)$ and $\left(\sigma_{1,2}, \hat{\delta}_{1}\right)$, respectively, which causes $\delta_{1}$ to be a leaf node adjacent to $\sigma$.

After insertion of the edge $\left(\sigma, \delta_{2}\right)$ the remaining edge of the form $\left(\sigma_{2, n}, \delta_{2}\right)$ is removed the same way. Since $\delta_{3}$ is already a leaf node, the insertion of $\left(\sigma, \delta_{3}\right)$ will cause it to be a leaf node adjacent to $\sigma$. 


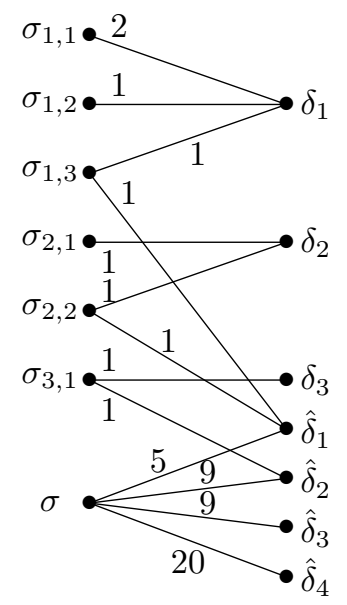

Figure 6 . The support graph $B(y)$ of the vertex $y$ of a transportation polytope $P$

To prove that the Hirsch Conjecture is true for transportation polytopes, one would hope that any pair of vertices that differ in $k$ support elements has a pivot step that reduces the number of non-zero variables in which the vertices differ, but Brightwell et al. 22] noticed that this was not true. We show their counter-example in Figure 7
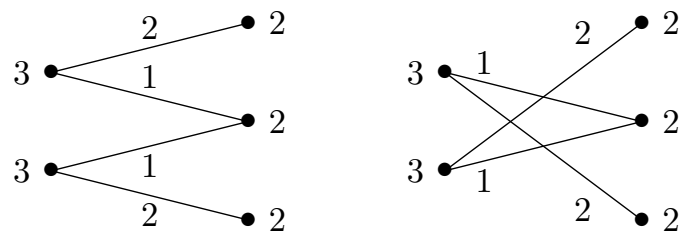

FiguRE 7. Support graphs of a pair of vertices where no pivot reduces the difference in support

Open Problem 2.23. Prove or disprove the Hirsch Conjecture for 2-way transportation polytopes.

By Corollary 2.18, this would mean the diameter is less than or equal to $p+q-1$. This conjecture holds for many special cases that restrict the margins. For example the conjecture is true for Birkhoff's polytope and for some special right-hand sides (see e.g., 20]).

While transportation polytopes seem tame compared to other polytopes. It has been shown that they have some non-trivial topological structure: Diameter bounds for simple $d$-polyhedra can be studied via decomposition properties of related simplicial complexes. Each non-degenerate simple polytope has a polar simplicial complex, a simplicial polytope. Billera and Provan (see [126]) showed that polytopes 
whose dual simplicial polytope is weakly vertex decomposable have a linear diameter. But it has recently been shown (see [59]) that the infinite family of polars of $p \times 2$ transportation polytopes for $p \geq 5$ are not weakly vertex-decomposable, the first ever such examples. But at the same time, one can prove the Hirsch Conjecture holds for $p \times 2$ transportation polytopes by proving a stronger statement:

Theorem 2.24. The Hirsch Conjecture holds for all convex polytopes obtained as the intersection of a cube and a hyperplane.

Fix a dimension $d \in \mathbb{N}$. Let $H=\left\{x \in \mathbb{R}^{d} \mid a_{1} x_{1}+\cdots+a_{d} x_{d}=b\right\}$ be the hyperplane determined by the non-zero normal vector $a=\left(a_{1}, \ldots, a_{d}\right)$ and $\mathrm{t}$ he constant $b \in \mathbb{R}$. Let $\square_{d}$ denote $d$-dimensional cube with 0 - 1 vertices. Then, let $P$ denote the polytope obtained as their intersection $P=\square_{d} \cap H$.

If the dimension of the polytope $P$ is less than $d-1$, then $P$ is a face of $\square_{d}$. In that case, $P$ itself is a cube of lower dimension, so we assume that the polytope $P$ is of dimension $d-1$. We may also assume that the polytope $P$ is not a facet of the $d$-cube, so that $H$ intersects the relative interior of $\square_{d}$.

Without assuming any genericity, a simple dimension argument shows that the vertices of the polytope $P$ are either on the relative interior of an edge of the cube $\square_{d}$ or are vertices of the cube. We assume that $H$ is sufficiently generic. Then, no vertex of the cube $\square_{d}$ will be a vertex of $P$. For each vertex $v$ of $P$, we define its side signature $\sigma(v)$ to be a string of length $d$ consisting of the characters $*, 0$, and 1 by the following rule:

$$
\sigma(v)_{i}= \begin{cases}0 & \text { if } v_{i}=0 \\ 1 & \text { if } v_{i}=1 \\ * & \text { if } 0<v_{i}<1\end{cases}
$$

By genericity, it cannot be the case that there are two vertices of $P$ with the same side signature. Indeed, if there were two distinct vertices $v$ and $w$ with the same side signature, then $P$ will contain the entire edge of the cube containing them both, and $v$ and $w$ will not be vertices.

Let $H_{i, 0}$ denote the hyperplane $\left\{x \in \mathbb{R}^{d} \mid x_{i}=0\right\}$ and let $H_{i, 1}$ denote the hyperplane $\left\{x \in \mathbb{R}^{d} \mid x_{i}=1\right\}$. If there is an $i \in[d]$ such that the hyperplane $H$ does not intersect $H_{i, 0}$ nor $H_{i, 1}$, then we can project $P$ to a lower-dimensional face of $I_{d}$. Thus, for each $i \in[d]$, we can assume that $H$ inte rsects at least one of $H_{i, 0}$ or $H_{i, 1}$.

Given two vertices $v=\left(v_{1}, \ldots, v_{d}\right)$ and $w=\left(w_{1}, \ldots, w_{d}\right)$ of $P=I_{d} \cap H$, we define the Hamming distance between them based on their side signatures:

$$
\operatorname{hamm}(v, w)=\sum_{i=1}^{d} \operatorname{hamm}\left(\sigma(v)_{i}, \sigma(w)_{i}\right),
$$

where

$\operatorname{hamm}(0,1)=\operatorname{hamm}(1,0)=\operatorname{hamm}(1, *)=\operatorname{hamm}(*, 1)=\operatorname{hamm}(0, *)=\operatorname{hamm}(*, 0)=1$

and

$$
\operatorname{hamm}(0,0)=\operatorname{hamm}(1,1)=\operatorname{hamm}(*, *)=0 .
$$

Lemma 2.25. Let $P$ defined as above using a sufficiently-generic hyperplane $H$. Let $v$ and $w$ be two vertices of $P$. Let $f(P)$ denote the number of facets of $P$. 
If $v$ and $w$ have the $*$ in the same coordinate and $P$ does not intersect either of the two facets in that direction, i.e., there is an $i$ such that $\sigma(v)_{i}=*=\sigma(w)_{i}$ and $P \cap H_{i, 1}=\emptyset=P \cap H_{i, 0}$, then $f(P) \geq(d-1)+\operatorname{hamm}(v, w)-1$.

Otherwise, $f(P) \geq(d-1)+\operatorname{hamm}(v, w)$.

Proof. By rotating the (combinatorial) cube if necessary, we can assume without loss of generality that the side signature $\sigma(v)$ of the vertex $v$ is $(*, 0,0, \ldots, 0)$ and that the side signature $\sigma(w)$ of the vertex $w$ is either of the form $(0, *, 0,0, \ldots, 0,1,1, \ldots, 1)$ with $k \geq 0$ trailing ones or of the form $(*, 0,0, \ldots, 0,1,1, \ldots, 1)$ with $k \geq 1$ trailing ones, after applying a suitable rotation to the cube.

In the first case, $\operatorname{hamm}(v, w)=k+1$ and we have at least $d$ " 0 -facets" and $k$ "1-facets."

In the second case, we have $d-1$ " 0 -facets", $k$ " 1 -facets" and (unless there is an $i$ such that $\sigma(v)_{i}=*=\sigma(w)_{i}$ and $\left.P \cap H_{i, 1}=\emptyset=P \cap H_{i, 0}\right)$ at least one more facet. Thus, $f(P) \geq(d-1)+k+1=(d-1)+\operatorname{hamm}(v, w)$, unless we are in the special case, in which case $f(P) \geq(d-1)+k+1-1$.

Lemma 2.26. Let $P$ defined as above using a sufficiently-generic hyperplane $H$. Let $v$ and $w$ be two vertices of $P$. Then, there is a pivot from the vertex $v$ to a vertex $v^{\prime}$ with $\operatorname{hamm}\left(v^{\prime}, w\right)=\operatorname{hamm}(v, w)-1$.

Proof. Again by rotating if necessary, without loss of generality, we can assume that $\sigma(v)=(*, 0,0, \ldots, 0)$ and that $\sigma(w)$ is either $(*, 1,1, \ldots, 1)$ or $(1,1, \ldots, 1, *)$.

If the side signature $\sigma(w)$ of $w$ is $(*, 1,1, \ldots, 1)$, performing a pivot on the vertex $v$ in any one of the $d-1$ last coordinates reduces the Hamming distance.

Otherwise, the side signature $\sigma(w)$ of $w$ is $(1,1, \ldots, 1, *)$. We now describe what can occur when pivoting from the vertex $v$ to a new vertex $v^{\prime}$. We claim that at least one of the $d-1$ possible pivots on the vertex $v$ does not put a 0 in the first coordinate of the side signature $\sigma\left(v^{\prime}\right)$ of the new vertex $v^{\prime}$. Otherwise, the hyperplane $H$ cuts the polytope $P$ as a vertex figure: that is to say, the polytope $P$ cuts the corner $(1,0, \ldots, 0)$ of the cube. See Figure 8 for a picture.

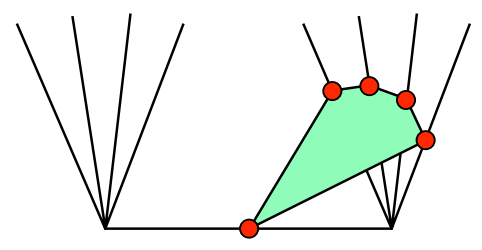

Figure 8 . The vertex $v$ on the horizontal axis (the $x_{1}$ coordinate increases moving to the right) and its neighboring vertices on orthogonal edges of the cube.

The remaining kind of pivots on $v$ that result in a new vertex $v^{\prime}$ give side signatures $\sigma\left(v^{\prime}\right)$ of one of the following three forms:

(1) The signature $\sigma\left(v^{\prime}\right)$ of the neighbor $v^{\prime}$ of the vertex $v$ could be

$$
(1,0,0, \ldots, *, 0, \ldots, 0),
$$

which reduces the Hamming distance by one. 
(2) The signature $\sigma\left(v^{\prime}\right)$ of the neighbor $v^{\prime}$ of the vertex $v$ could be

$$
(*, 0,0, \ldots, 0,1,0,0, \ldots, 0),
$$

which reduces the Hamming distance by one.

(3) Otherwise, one remaining pivot could give the side signature

$$
(1,0,0, \ldots, 0, *)
$$

for $\sigma\left(v^{\prime}\right)$.

This third type of pivot does not reduce the Hamming distance. But if this is the only pivot that could give this and the first two kinds of pivots cannot be performed, then all of the remaining pivots are the kind that put 0 in the first coordinate of the side signature $\sigma(v)$ of $v$. But this would imply that $H$ could not have intersected the hyperplane $H_{d+}$, and thus $P$ would be a $(d-1)$-cube with one vertex truncated.

Corollary 2.27. Let $P \neq \emptyset$ be a classical transportation polytope of size $p \times 2$ with $n \leq 2 p$ facets. Then, the dimension of $P$ is $d=p-2$ and the diameter of $P$ is at most $n-d$.

To see this follows from the previous theorem, we note that the coordinateerasing projection of $P$ to the coordinates $x_{1,1}, x_{2,1}, \ldots, x_{p, 1}$ of the first column shows that $P$ is the intersection of a hyperplane with a rectangular prism. (In particular, if the intervals are all equal and one has a cube, then the Minkowski sum of two consecutive hypersimplices $D(p, i)$ and $D(p, i+1)$ can be realized as a transportation polytope of size $p \times 2$.) After an affine transformation, the polytope $P$ is the intersection of a hyperplane and a cube. (The transformation takes the cube $\left[0, u_{1}\right] \times \cdots \times\left[0, u_{p}\right]$ to the cube $[0,1]^{p}$. That is to say, the $i$ th coordinate $y_{i}$ in the cube $[0,1]^{p}$ is $x_{i, 1} / u_{i}$.) By applying an affine transformation to $P=\square_{d} \cap H$, we obtain a $p \times 2$ classical transportation polytope.

The Hirsch bound also holds for Birkhoff polytopes:

Theorem 2.28. Let $B_{p}$ be the pth Birkhoff polytope then

(1) the degree of each vertex of $B_{p}$ is

$$
\sum_{k=0}^{p-2}\left(\begin{array}{l}
p \\
k
\end{array}\right)(p-k-1) !
$$

(2) If $p \geq 4$, the diameter of $B_{p}$ is 2 .

(3) (Billera-Sarangarajan [16]) Every pair of vertices $x, y$ is contained in a cubical face. The dimension of this cubical face is the number of cycles in the union of $B(x)$ and $B(y)$.

Proof. For part 1. note that because the symmetric group acts transitively on the vertices (which are permutation matrices) the degree of all vertices is the same. It suffices to count how many vertices are adjacent to the vertex corresponding to the identity matrix. Any adjacent vertex $y$ has $k$ common edges with $x$ for $k=0, \ldots, p-2$. Now the $k$ edges can be chosen in $\left(\begin{array}{l}p \\ k\end{array}\right)$ ways and for each choice we have a unique cycle being formed with the remaining $(p-k-1)$ pairs of vertices $\left(i, i^{\prime}\right)$. This can be done in $(p-k-1)$ ! ways.

Now we prove part 2 Given two non-adjacent vertices $x$ and $y$ we have a third vertex $z$ adjacent to both. Without loss of generality, the two graphs $B(x)$ 
and $B(y)$ have no common edges, otherwise apply induction. Thus they define $p$ disjoint bipartite cycles, as shown in Figure 9.

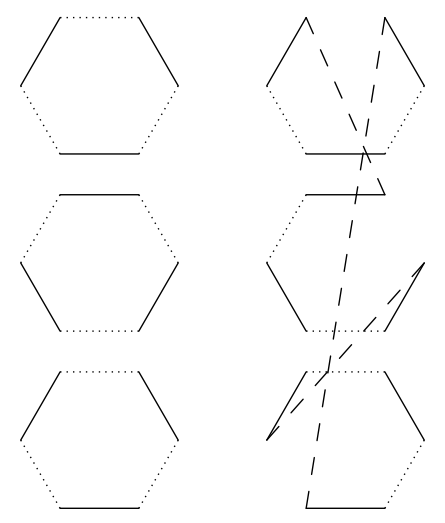

FIGURE 9. Diameter of Birkhoff polytope

It is worth noting that even if the Hirsch Conjecture for transportation polytopes is true, the simplex method may behave badly because there could be long decreasing pivot sequences:

Theorem 2.29 (I. Pak [122]). Consider the linear functional

$$
c_{\alpha} \cdot x=x_{1,1}+\alpha x_{1,2}+\cdots+\alpha^{p-1} x_{1, p}+\alpha^{p} x_{2,1}+\cdots+\alpha^{p^{2}-1} x_{p, p} .
$$

For $1 / p>\alpha>0$, there exist a decreasing sequence of vertices of the $p \times p$ Birkhoffvon Neumann polytope of length $K p$ ! for a universal constant $K$.

However, Pak (see [122]) also showed the more encouraging result that the expected average running time of the simplex method on the Birkhoff polytope with cost vector $c_{\alpha}$ is $O(p \log p)$.

2.3. Integer points. Questions on the integer, or lattice, points of transportation polytopes are very popular in combinatorics. Objects such as magic squares, magic labelling of graphs and sudoku arrangements can be presented as lattice points of transportation polytopes. See, for instance, [14, 54, 135] and the references therein. How many ways are there to fill the entries of a $p \times q$ table with margins $u$ and $v$ using only non-negative integer entries $x_{i, j}$ ? E.g., see Figure 10 . This counting problem is a \#P-complete problem, even for $2 \times q$ tables (see [7]).

The lattice points of dilations of the Birkhoff polytope are called semi-magic squares: that is to say, a semi-magic square is an integral lattice point in a transportation polytope where every row and column sum is the same, namely $\zeta$. The number $\zeta$ is called the magic number. Counting these objects is a rather natural combinatorial problem that has been studied by many researchers. In 60, De Loera, Liu, and Yoshida presented a generating function for the number of semimagic squares and formulas for the coefficients of the Ehrhart polynomial of the $p$ th Birkhoff polytope $B_{p}$. In particular they also deduced a combinatorial formula for the volume of Birkhoff polytopes. The volume formula is a multivariate generating 


\begin{tabular}{|c|c|c|c|c}
\hline 68 & 119 & 26 & 7 & 220 \\
\cline { 1 - 4 } 20 & 84 & 17 & 94 & 215 \\
\cline { 1 - 3 } 15 & 54 & 14 & 10 & 93 \\
\cline { 1 - 3 } 5 & 29 & 14 & 16 & 64 \\
108 & 286 & 71 & 127
\end{tabular}

There are $1,225,914,276,768,514$ such tables.

FiguRE 10. This transportation polytope has many lattice points!

function for the lattice points of the Birkhoff polytope and all its dilations. Unfortunately the number of terms, which alternate in sign, is quite large. The summation runs over all the possible arborescences of a complete graph in $p$ nodes $\left(p^{p-2}\right.$ of them) and the $p$ ! permutations, thus the formula is quite large and not efficient to evaluate. The key elements of this formula come from understanding triangulations of the tangent cones of the Birkhoff polytope and the algorithmic theory of lattice points developed by Barvinok (see [11, 12]). More recently Liu (see 110]) described the same kind of generating functions for perturbations of the Birkhoff polytope into simple transportation polytopes (i.e., the margin sum conditions are not one but a small change in value). She obtained similar combinatorial formulas for the generalized Birkhoff $k p \times p$ polytope. She also recovered the formula for the maximum possible number of vertices of transportation polytopes of order $k p \times p$ that had been studied in the literature before. Prior work on enumeration includes [36, where Carlitz described lattice points of dilations of the Birkhoff polytope using exponential generating functions.

Counting magic squares and lattice points in (dilations of) Birkhoff polytopes is related to computing their volumes. The computation of volumes and triangulations of the Birkhoff polytope is related to the problem of generating a random doubly stochastic matrix (see [37). The volume problem has been studied by many researchers (see 11, 2, 14, 15, 37, 64, 88, 92, 122, 134, 135, among others). The exact value of the volume of the $p$ th Birkhoff polytope $B_{p}$ is known (see [125]) only up to $p=10$. Canfield and McKay (see [35) presented an asymptotic formula for the volume of the $p$ th Birkhoff polytope $B_{p}$. In [10, Barvinok also presented asymptotic upper and lower bounds for the volumes of $p \times q$ classical transportation polytopes and the number of $p \times q$ semi-magic rectangles.

The currently known exact values of $a(p)$ are summarized in Table 2 .

To compute more values it would useful to know the answer to the following problem:

Open Problem 2.30. Is there a short (polynomial time computable) formula for the normalized volume $a(p)$ of the $p \times p$ Birkhoff-von Neumann polytope?

Besides knowing the volumes and the number of vertices, we are interested in knowing the so-called integer range of a coordinate in a transportation polytope $P$. This asks the following: fixing $i$ and $j$, do all integers in an interval appear as the value of the coordinate $x_{i, j}$ of among the set of lattice points of $P$ ? For classical transportation polytopes, the answer is yes: 


\begin{tabular}{|c|l|}
\hline$p$ & $a(p)$ \\
\hline 1 & 1 \\
\hline 2 & 1 \\
\hline 3 & 3 \\
\hline 4 & 352 \\
\hline 5 & 4718075 \\
\hline 6 & 14666561365176 \\
\hline 7 & 17832560768358341943028 \\
\hline 8 & 12816077964079346687829905128694016 \\
\hline 9 & 7658969897501574748537755050756794492337074203099 \\
\hline 10 & 5091038988117504946842559205930853037841762820367901333706255223000 \\
\hline
\end{tabular}

TABLE 2. Normalized volumes of Birkhoff polytopes

Lemma 2.31 (Diaconis and Gangolli 65, Integer range of a coordinate). For an entry $x_{i, j}$ of the transportation polytope with marginals $u$ and $v$, the set of all possible integral values are the integers on a segment.

This gives a method of performing the so-called sequential importance sampling (see, e.g., [40, 145]). Chen et al. (see [40]) use the interval property to justify correctness of their algorithm for the sequential sampling of entries in multi-way contingency tables with given constraints. This method of sampling contingency tables with given margins introduced in 39 is later extended by Chen in 38 to sample tables with fixed marginals and a given set of structural zeros.

For many applications, again including sampling and enumerating lattice points, we are interested in having a set of "local moves" or operations that connect the set of all integer contingency tables with fixed margins. E.g., such a set of moves is important in probability and statistics in the interest of running Markov chains on contingency tables (see [61]). As it turns out the set of moves necessary is quite simple:

Lemma 2.32. The set of "rectangular" vectors whose entries are $0,-1$, and 1 (as in Figure 11) corresponding to 4-cycles in the complete bipartite graph $K_{p, q}$, with $a 1$ and $a-1$ in each row column are integer vectors in the kernel of the constraint matrix of 2-way transportation polytopes. They are simple moves that connect all lattice points of any 2-way transportation polytope.

\begin{tabular}{|c|c|c|c|c|c|c|c|c|}
\hline 0 & 0 & 0 & 0 & 0 & 0 & 0 & 0 & 0 \\
\hline 0 & 0 & -1 & 0 & 0 & 0 & 1 & 0 & 0 \\
\hline 0 & 0 & 0 & 0 & 0 & 0 & 0 & 0 & 0 \\
\hline 0 & 0 & 0 & 0 & 0 & 0 & 0 & 0 & 0 \\
\hline 0 & 0 & 1 & 0 & 0 & 0 & -1 & 0 & 0 \\
\hline 0 & 0 & 0 & 0 & 0 & 0 & 0 & 0 & 0 \\
\hline 0 & 0 & 0 & 0 & 0 & 0 & 0 & 0 & 0 \\
\hline
\end{tabular}

Figure 11. Typical monomial in Graver basis

Using these moves one can run a Markov chain on all the vertices of a transportation polytope, where we move from one vertex to another by adding one of the 
randomly generated moves that preserves non-negativity. Cryan et al. (see 49]) have shown that the associated Markov chain mixes rapidly when the number $p$ or $q$ of rows or columns is assumed fixed.

This set of vectors is an example of a Graver basis for the kernel of the matrix associated to the 2-way transportation polytope in question (see 83]). Formally, to define a Graver basis, we first describe a partial order $\sqsubseteq$ on $\mathbb{Z}^{n}$. Given two integer vectors $u, v \in \mathbb{Z}^{n}$, we say $u \sqsubseteq v$ if $\left|u_{k}\right| \leq\left|v_{k}\right|$ and $u_{k} v_{k} \geq 0$ for all $k=1, \ldots, n$. Then the Graver basis of a matrix $A$ is the set of all $\sqsubseteq$-minimal vectors in $\left\{x \in \mathbb{Z}^{n} \mid\right.$ $A x=0, x \neq 0\}$. Graver bases are quite important in optimization (see Chapters 3 and 4 of [55] and the nice book [121] for details).

In the next section, we discuss multi-way transportation polytopes. As we will see, their behavior is much more complicated.

\section{Multi-WAy transportation polytopes}

Classical transportation polytopes were called 2-way transportation polytopes because the coordinates $x_{i, j}$ have two indices. We can consider generalizations of 2-way transportation polytopes by having coordinates indexed by three or more integers (e.g., $x_{i, j, k}$ or $x_{i, j, k, l}$ ). As the number of indices grows the possible form and shape of constraints grows. There has been very active work on understanding the corresponding polyhedra (see e.g., [86, 87, 115, 116, 129, 131, 132, 133, 141). As we will see here the case of 3-way transportation problems, i.e., three indices, is already so complicated that in a sense contains all polyhedral geometry and combinatorial optimization!

A $d$-way table of size $p_{1} \times \cdots \times p_{d}$ is a $p_{1} \times p_{2} \times \cdots \times p_{d}$ array of non-negative real numbers $x=\left(x_{i_{1}, \ldots, i_{d}}\right), 1 \leq i_{\ell} \leq p_{\ell}$. Given an integer $m$, with $0 \leq m<d$, an $m$-margin of the $d$-way table $x$ is one of the $\left(\begin{array}{c}d \\ m\end{array}\right)$ possible $m$-tables obtained by summing the entries over all but $m$ indices. For example, if $\left(x_{i, j, k}\right)$ is a 3 -way table then its 0-marginal is $x_{+,+,+}=\sum_{i=1}^{p_{1}} \sum_{j=1}^{p_{2}} \sum_{k=1}^{p_{3}} x_{i, j, k}$, it has three 1-margins, which are $\left.x_{i,+,+}\right)=\sum_{j=1}^{p_{2}} \sum_{k=1}^{p_{3}} x_{i, j, k}$ and likewise $\left(x_{+, j,+}\right),\left(x_{+,+, k}\right)$. Finally $x$ has three 2-margins given by the sums $\left(x_{i, j,+}\right)=\sum_{k=1}^{p_{3}} x_{i, j, k}$ and likewise $\left(x_{i,+, k}\right)$, $\left(x_{+, j, k}\right)$.

A $d$-way transportation polytope of size $p_{1} \times \cdots \times p_{d}$ defined by $m$-marginals is the set of all $d$-way tables of size $p_{1} \times p_{2} \times \cdots \times p_{d}$ with the specified marginals. When $d=2$, we recover the classical transportation polytopes of the previous section. When $d \geq 3$, the transportation polytope is also called a multi-way transportation polytope. When $d=3$, we will typically denote the size of the transportation polytope by $p \times q \times s$ instead of $p_{1} \times p_{2} \times p_{3}$.

In a well-defined sense the most important margins of a $d$-way transportation polytope are the $(d-1)$-margins:

Theorem 3.1 (Junginger 99]). There exists a polynomial time algorithm that, given a linear (integer) minimization problem over a $d$-way $p_{1} \times \cdots \times p_{d}$ transportation polytope $T_{d, m}$ with fixed $m$-marginals and cost vector $c$, computes an associated linear functional $\hat{c}$ and a $d$-way $\left(p_{1}+1\right) \times \cdots \times\left(p_{d}+1\right)$ transportation polytope $T_{d, d-1}$ with fixed $(d-1)$-marginals such that if $y$ is an optimal (integral) solution for $T_{d, d-1}$ its entries with indices with the original range also give an optimal (integral) solution of $T_{d, m}$. 
Example 3.2. We illustrate Junginger's theorem in the 3-way case. Suppose we have a linear optimization problem over a 3-way $p \times q \times s$ transportation defined by 1-marginals:

$$
\begin{array}{ll}
\operatorname{minimize} & \sum_{i_{1}=1}^{p} \sum_{i_{2}=1}^{q} \sum_{i_{3}=1}^{s} c_{i_{1}, i_{2}, i_{3}} x_{i_{1}, i_{2}, i_{3}} \\
\text { subject to }\left\{\begin{array}{l}
\sum_{i_{2}=1}^{q} \sum_{i_{3}=1}^{s} x_{i_{1}, i_{2}, i_{3}}=b_{i_{1},+,+} \\
\sum_{i_{1}=1}^{p} \sum_{i_{3}=1}^{s} x_{i_{1}, i_{2}, i_{3}}=b_{+, i_{2},+} \\
\sum_{i_{1}=1}^{p} \sum_{i_{2}=1}^{q} x_{i_{1}, i_{2}, i_{3}}=b_{+,+, i_{3}} \\
x_{i_{1}, i_{2}, i_{3}} \geq 0
\end{array}\right.
\end{array}
$$

Junginger showed this can be solved instead using a 3 -way $(p+1) \times(q+1) \times(s+1)$ transportation polytope with fixed 2-marginals:

$$
\begin{array}{ll}
\text { minimize } & \sum_{i_{1}=1}^{p+1} \sum_{i_{2}=1}^{q+1} \sum_{i_{3}=1}^{s+1} \hat{c}_{i_{1}, i_{2}, i_{3}} y_{i_{1}, i_{2}, i_{3}} \\
\text { subject to }\left\{\begin{array}{l}
\sum_{i_{1}=1}^{p+1} y_{i_{1}, i_{2}, i_{3}}=a_{+, i_{2}, i_{3}}, \\
\sum_{i_{2}=1}^{q+1} y_{i_{1}, i_{2}, i_{3}}=a_{i_{1},+, i_{3}}, \\
s+1 \\
\sum_{i_{3}=1}^{s+1} y_{i_{1}, i_{2}, i_{3}}=a_{i_{1}, i_{2},+}, \\
y_{i_{1}, i_{2}, i_{3}} \geq 0 .
\end{array}\right.
\end{array}
$$

Here the cost coefficients $\hat{c}_{i_{1}, i_{2}, i_{3}}$ and the 2-marginals $a$ are as follows:

$$
\hat{c}_{i_{1}, i_{2}, i_{3}}= \begin{cases}c_{i_{1}, i_{2}, i_{3},}, & \text { if all } 3 \text { indices are within the original ranges } \\ M, & \text { if exactly } 2 \text { of the indices are within the original range } \\ 0, & \text { otherwise. }\end{cases}
$$

Let $\beta=\max \left(b_{i_{1},+,+}, b_{+, i_{2},+}, b_{+,+, i_{3}}\right)$.

When $i_{1}, i_{2}, i_{3}$ stay within the original ranges:

$$
a_{+, i_{2}, i_{3}}=\beta ; \quad a_{i_{1},+, i_{3}}=\beta ; \quad a_{i_{1}, i_{2},+}=\beta .
$$

When we go outside the ranges in exactly one of the indices:

$$
\begin{array}{ll}
a_{+, q+1, i_{3}}=p \beta-b_{+,+, i_{3}}, & a_{+, i_{2}, s+1}=p \beta-b_{+, i_{2},+}, \\
a_{i_{1},+, s+1}=q \beta-b_{i_{1},+,+}, & a_{p+1,+, i_{3}}=q \beta-b_{+,+, i_{3}}, \\
a_{p+1, i_{2},+}=s \beta-b_{+, i_{2},+}, & a_{i_{1}, q+1,+}=s \beta-b_{i_{1},+,+} .
\end{array}
$$

Finally, when exactly two of the indices are outside the original range:

$$
a_{+, q+1, s+1}=a_{p+1,+, s+1}=a_{p+1, q+1,+}=\beta .
$$

Now for each solution $x_{i_{1}, i_{2}, i_{3}}$ of the 3 -way problem with 1-marginals we can recover a unique solution $y_{i_{1}, i_{2}, i_{3}}$ of the 3 -way problem with 2-marginals that has the same objective function value plus a constant. If $x$ is integral, then $y$ will be 
integral too when the marginals have integral entries. For this set the value of $y_{i_{1}, i_{2}, i_{3}}:=x_{i_{1}, i_{2}, i_{3}}$ when all $i_{\ell}$ are in the original range. Using the new 2-marginal equations determine the values of those variables $y_{i_{1}, i_{2}, i_{3}}$ with exactly one index outside original range. Thus for fixed $i_{2}, i_{3}$ in the original range:

$$
y_{p+1, i_{2}, i_{3}}=a_{+, i_{2}, i_{3}}-\sum_{i_{1}=1}^{p} y_{i_{1}, i_{2}, i_{3}}=\beta \sum_{i_{1}=1}^{p} x_{i_{1}, i_{2}, i_{3}} \geq 0 .
$$

Next fill the values of those variables $y_{i_{1}, i_{2}, i_{3}}$ with exactly two indices outside range. Finally fill the variable $y_{p+1, q+1, s+1}$. It is easy (but tedious) to check that $y_{i_{1}, i_{2}, i_{3}}$ is indeed feasible in the 2-marginal problem.

Now the objective function value is

$$
\begin{gathered}
\sum_{i_{1}=1}^{p+1} \sum_{i_{2}=1}^{q+1} \sum_{i_{3}=1}^{s+1} \hat{c}_{i_{1}, i_{2}, i_{3}} y_{i_{1}, i_{2}, i_{3}}=\sum_{i_{1}=1}^{p} \sum_{i_{2}=1}^{q} \sum_{i_{3}=1}^{s} c_{i_{1}, i_{2}, i_{3}} x_{i_{1}, i_{2}, i_{3}}+ \\
M\left(\sum_{i_{1}=1}^{p} x_{i_{1}, q+1, s+1}+\sum_{i_{2}=1}^{q} x_{p+1, i_{2}, s+1}+\sum_{i_{3}=1}^{s} x_{p+1, q+1, i_{3}}\right)
\end{gathered}
$$

which is equal to

$$
\sum_{i_{1}=1}^{p} \sum_{i_{2}=1}^{q} \sum_{i_{3}=1}^{s} c_{i_{1}, i_{2}, i_{3}} x_{i_{1}, i_{2}, i_{3}}+3 M \beta
$$

Conversely, if $y$ is the optimal solution for the 2-marginal problem, the restriction $x$ to those variables with indices $i_{1} \leq p, i_{2} \leq q, i_{3} \leq s$ is an optimal solution of the 1-marginal problem. For this note that because $y$ is optimal the entries of variables with two indices above the original range (e.g. $y_{p+1, q+1, i_{3}}$ ) must be zero because their cost is $M$ (a huge constant). Next check $x_{i_{1}, i_{2}, i_{3}}$ is feasible for the 1-marginal problem. Non-negativity is easy: note that

$$
\sum_{i_{2}=1}^{q} y_{p+1, i_{2}, i_{3}}=\sum_{i_{2}=1}^{q}\left(a_{+, i_{2}, i_{3}}-\sum_{i_{1}=1}^{p} y_{i_{1}, i_{2}, i_{3}}\right)=q \beta-b_{+,+, i_{3}} .
$$

Therefore, for the 1-marginal $b_{+,+, i_{3}}$,

$\sum_{i_{1}=1}^{p} \sum_{i_{2}=1}^{q} y_{i_{1}, i_{2}, i_{3}}=\sum_{i_{1}=1}^{p+1} \sum_{i_{2}=1}^{q} y_{i_{1}, i_{2}, i_{3}}-\sum_{i_{2}=1}^{q} y_{p+1, i_{2}, i_{3}}=q \beta-\left(q \beta-b_{+,+, i_{3}}\right)=b_{+,+, i_{3}}$. and the same can be checked for other 1-marginals.

Depending on the application a transportation problem may have a combination of margins that define polyhedron.

For 3-way transportation problems there are two natural generalization of 2-way transportation polytopes to 3-way transportation polytopes, whose feasible points are $p \times q \times s$ tables of non-negative reals satisfying certain sum conditions:

- First, consider the 3-way transportation polytope of size $p \times q \times s$ defined by 1-marginals: Let $u=\left(u_{1}, \ldots, u_{p}\right) \in \mathbb{R}^{p}, v=\left(y_{1}, \ldots, y_{q}\right) \in \mathbb{R}^{q}$, and $w=\left(w_{1}, \ldots, w_{s}\right) \in \mathbb{R}^{s}$ be three vectors. Let $P$ be the polyhedron defined by the following $p+q+s$ equations in the pqs variables $x_{i, j, k} \in \mathbb{R}_{\geq 0}$ $(i \in[p], j \in[q], k \in[s])$ :

$$
\sum_{j, k} x_{i, j, k}=u_{i}, \forall i \quad \sum_{i, k} x_{i, j, k}=v_{j}, \forall j \quad \sum_{i, j} x_{i, j, k}=w_{k}, \forall k .
$$


In [146, 3-way transportation polytopes defined by all 1-marginals are known as 3-way axial transportation polytopes.

- Similarly, a 3-way transportation polytope of size $p \times q \times s$ can be defined by specifying three real-valued matrices $U, V$, and $W$ of sizes $q \times s, p \times s$, and $p \times q$ (respectively). These three matrices specify the line-sums resulting from fixing two of the indices of entries and adding over the remaining index. That is to say, the polyhedron $P$ is defined by the following $p q+p s+q s$ equations, the 2-marginals, in the $p q s$ variables $x_{i, j, k} \in \mathbb{R}_{\geq 0}$ satisfying:

$$
\sum_{i} x_{i, j, k}=U_{j, k}, \forall j, k \quad \sum_{j} x_{i, j, k}=V_{i, k}, \forall i, k \quad \sum_{k} x_{i, j, k}=W_{i, j}, \forall i, j .
$$

In [146], the 3-way transportation polytopes defined by 2-marginals are called 3-way planar transportation polytopes.

3.1. Why $d$-way transportation polytopes are harder. The 3-way transportation polytopes are very interesting because of the following universality theorem of De Loera and Onn in 62 which says that for any rational convex polytope $P$, there is a 3 -way planar transportation polytope $T$ isomorphic to $P$ in a very strong sense.

We say a polytope $P \subset \mathbb{R}^{p}$ is representable as a polytope $T \subset \mathbb{R}^{q}$ if there is an injection $\sigma:\{1, \ldots, p\} \longrightarrow\{1, \ldots, q\}$ such that the projection $\pi: \mathbb{R}^{q} \longrightarrow \mathbb{R}^{p}$

$$
x=\left(x_{1}, \ldots, x_{q}\right) \mapsto \pi(x)=\left(x_{\sigma(1)}, \ldots, x_{\sigma(p)}\right)
$$

is a bijection between $T$ and $P$ and between the sets of integer points $T \cap \mathbb{Z}^{q}$ and $P \cap \mathbb{Z}^{p}$.

Note that if $P$ is representable as $T$ then $P$ and $T$ have same facial structure and all linear or integer programming programs are polynomial-time equivalent. We can state the universality result as follows:

Theorem 3.3 (Universality [62]). Any polytope $P=\left\{y \in \mathbb{R}_{\geq 0}^{n}: A y=b\right\}$ with integer $m \times n$ matrix $A=\left(a_{i, j}\right)$ and integer vector $b$ is polynomial-time representable as a slim $r \times c \times 3$ transportation polytope

$T=\left\{x \in \mathbb{R}_{\geq 0}^{r \times c \times 3}: \sum_{i} x_{i, j, k}=U_{j, k}, \sum_{j} x_{i, j, k}=V_{i, k}, \sum_{k} x_{i, j, k}=W_{i, j}\right\}$,

with $r=O\left(m^{2}(n+L)^{2}\right)$ rows and $c=O(m(n+L))$ columns, where $L:=\sum_{j=1}^{n} \max _{i=1}^{m}\left\lfloor\log _{2}\left|a_{i, j}\right|\right\rfloor$.

The constructive proof of Theorem 3.3 follows three steps.

(1) Decrease the size of the coefficients used in the constraints.

(2) Encode the polytope $P$ as a transportation polytope with 1-margins and with some entries bounded

(3) Encode any transportation polytope with 1-margins and bounded entries into a new transportation polytope with 2-margins

We only explain steps 1 and 2 which already give an interesting corollary.

Step 1: Given $P=\{y \geq 0: A y=b\}$ where $A=\left(a_{i, j}\right)$ is an integer matrix and $b$ is an integer vector. We represent it as a polytope $Q=\{x \geq 0: C x=d\}$, in polynomial-time, with a $\{-1,0,1,2\}$-valued matrix $C=\left(c_{i, j}\right)$ of coefficients. For this use the binary expansion $\left|a_{i, j}\right|=\sum_{s=0}^{k_{j}} t_{s} 2^{s}$ with all $t_{s} \in\{0,1\}$, we rewrite this term as $\pm \sum_{s=0}^{k_{j}} t_{s} x_{j, s}$. 
For example, the equation $3 y_{1}-5 y_{2}+2 y_{3}=7$ becomes

$$
\begin{aligned}
& \begin{array}{lll}
2 x_{1,0}-x_{1,1} & =0,
\end{array} \\
& \begin{array}{lll}
2 x_{2,0} & -x_{2,1} & = \\
& & 0
\end{array} \\
& \begin{array}{lll}
2 x_{2,1}-x_{2,2} & =0,
\end{array} \\
& \begin{array}{lllll}
x_{1,0} & +x_{1,1} & -x_{2,0} & -x_{2,2} & +x_{3,1}
\end{array}
\end{aligned}
$$

Step 2: Here is a sketch. Each equation $k=1, \ldots, m$ will be encoded in a "horizontal table" plus an extra layer of "slacks". Each variable $y_{j}, j=1, \ldots, n$ will be encoded in a "vertical box". Other entries are zero. See Figure 12 , Given $P=\{y \geq$

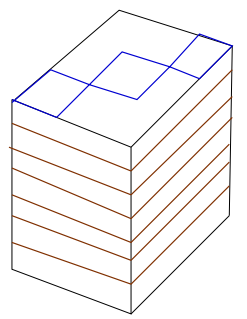

FiguRE 12. Each equation is encoded in one of the 6 horizontal tables, with the seventh table used for slacks

$0: A y=b\}$ where $A=\left(a_{i, j}\right)$ is an $m \times n$ integer matrix and $b$ is an integer vector: we assume that $P$ is bounded and hence a polytope, with an integer upper bound $U$ on the value of any coordinate $y_{j}$ of any $y \in P$. The sizes of the layers will be given by the numbers $r_{j}:=\max \left(\sum_{k}\left\{a_{k, j}: a_{k, j}>0\right\}, \sum_{k}\left\{\left|a_{k, j}\right|: a_{k, j}<0\right\}\right)$ and $r:=\sum_{j=1}^{n} r_{j}, R:=\{1, \ldots, r\}, m+1$ and $H:=\{1, \ldots, m+1\}$. Each equation $k=1, \ldots, m$ is encoded in a "horizontal table" $R \times R \times\{k\}$. The last horizontal table $R \times R \times\{m+1\}$ is included for consistency and its entries can be regarded as "slacks". Each variable $y_{j}, j=1, \ldots, n$ will be encoded in a "vertical box" $R_{j} \times R_{j} \times H$, where $R=\biguplus_{j=1}^{n} R_{j}$ is the natural partition of $R$ with $\left|R_{j}\right|=r_{j}$, namely with $R_{j}:=\left\{1+\sum_{l<j} r_{l}, \ldots, \sum_{l \leq j} r_{l}\right\}$.

For instance, if we have three variables, with $r_{1}=3, r_{2}=1, r_{3}=2$ then $R_{1}=$ $\{1,2,3\}, R_{2}=\{4\}, R_{3}=\{5,6\}$, and the top view of the matrix $x=\left(x_{i, j,+}\right)$ is

$$
\left(\begin{array}{cccccc}
x_{1,1,+} & x_{1,2,+} & 0 & 0 & 0 & 0 \\
0 & x_{2,2,+} & x_{2,3+} & 0 & 0 & 0 \\
x_{3,1,+} & 0 & x_{3,3,+} & 0 & 0 & 0 \\
0 & 0 & 0 & x_{4,4,+} & 0 & 0 \\
0 & 0 & 0 & 0 & x_{5,5,+} & x_{5,6,+} \\
0 & 0 & 0 & 0 & x_{6,5,+} & x_{6,6,+}
\end{array}\right)=\left(\begin{array}{cccccc}
y_{1} & \bar{y}_{1} & 0 & 0 & 0 & 0 \\
0 & y_{1} & \bar{y}_{1} & 0 & 0 & 0 \\
\bar{y}_{1} & 0 & y_{1} & 0 & 0 & 0 \\
0 & 0 & 0 & U & 0 & 0 \\
0 & 0 & 0 & 0 & y_{3} & \bar{y}_{3} \\
0 & 0 & 0 & 0 & \bar{y}_{3} & y_{3}
\end{array}\right) .
$$


The actual vertical position is decided with respect to the equations that contain a variable. Now, to specify the actual 1-margins: All "vertical" plane-sums are set to the same value $U$, that is, $u_{j}:=v_{j}:=U$ for $j=1, \ldots, r$. All entries not in the union $\biguplus_{j=1}^{n} R_{j} \times R_{j} \times H$ of the variable boxes will be forbidden. The horizontal plane-sums $w$ are determined as follows: For $k=1, \ldots, m$, consider the $k$ th equation $\sum_{j} a_{k, j} y_{j}=b_{k}$. Define the index sets $J^{+}:=\left\{j: a_{k, j}>0\right\}$ and $J^{-}:=\left\{j: a_{k, j}<0\right\}$, and set $w_{k}:=b_{k}+U \cdot \sum_{j \in J^{-}}\left|a_{k, j}\right|$.

Example 3.4. What do the three steps of this construction do, if one starts with the zero-dimensional polytope $P=\{y \mid 2 y=1, y \geq 0\}$ ? In this case, we obtain the 2 -margins of a 3-way transportation polytope shown in Figure 13 .

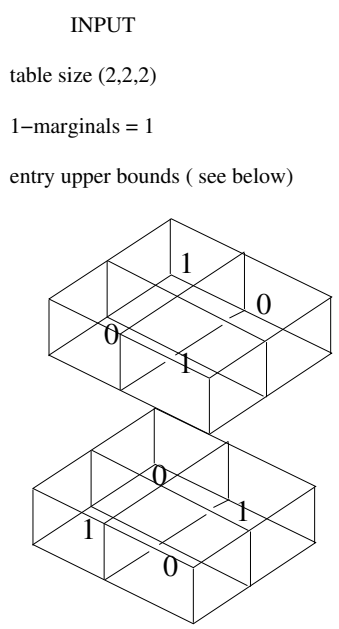

Unique real feasible array All entries $=1 / 2$ or 0

No integer table

\section{OUTPUT}

table size $(3,4,6)$

2-marginals (see below)

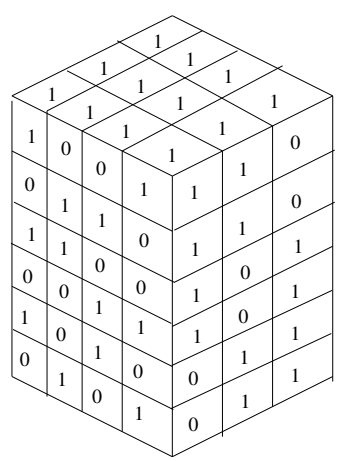

Unique real feasible array All entries $1 / 2$ and 0

No integer table.

FIGURE 13. Illustration of the universality theorem: A polyhedron consisting of the single point $y=\frac{1}{2}$ is represented by a $3 \times 4 \times 6$ polytope with 2-margins shown at the right of the figure. The left side of the figure shows the encoding using only the first two steps of the algorithm.

3.2. Comparing 2-way and 3-way transportation polytopes. We want to stress some consequences of the construction. First of all, simply from the first two steps above the following interesting theorem follows. Any rational polyhedron is a face of some axial 3-way transportation polytope.

Corollary 3.5. Any rational polytope $P=\left\{y \in \mathbb{R}^{n} \mid A y=b, y \geq 0\right\}$ is polynomialtime representable as a face of a 3-way $r \times c \times 3$ transportation polytope with 1-margins

$$
T=\left\{x \in \mathbb{R}_{\geq 0}^{r \times c \times 3}: \sum_{j, k} x_{i, j, k}=u_{i}, \sum_{i, k} x_{i, j, k}=v_{j}, \sum_{i, j} x_{i, j, k}=w_{k}\right\} .
$$


The properties we have seen in Section 2 for classical 2-way transportation polytopes raise the issue whether the analogous questions or properties hold for multiway transportation polytopes. We will address the following:

(1) Real feasibility (Vlach Problems [141]): Is there a simple characterization in terms of the 2-margins of those 3 -way transportation polytopes which are empty?

In particular, do any of the conditions on the margins proposed by Schell, Haley, Moravek and Vlack (see pages 374-376 of [146]) suffice to guarantee that the polytope is non-empty?

(2) Dimension: What are the possible dimensions of a $p \times q \times s$ transportation polytope? Is it always equal to $(p-1)(q-1)(s-1)$ ?

(3) Graphs of 3-way transportation polytopes: Do we have a good bound for the diameter? Is the Linear Hirsch Conjecture true in this case?

(4) Number of vertices of 3-way transportation polytopes: Can one estimate minimum and maximum number of vertices possible? Do they have a nice characterization?

(5) Integer Feasibility Problem: Given a prescribed collection of integral margins that seem to describe a $d$-way transportation polytope of size $p_{1} \times$ $\cdots \times p_{d}$, does there exist an integer table with these margins? Can such an integral $d$-way table be efficiently determined?

(6) Integer Range Property: Given a collection of margins coming from $d$-way table, and an index tuple $\left(i_{1}, \ldots, i_{d}\right)$, do all integer values inside the range of an interval appear for the coordinate $x_{i_{1}, \ldots, i_{d}}$ in the corresponding transportation polytope?

(7) Graver/Markov basis for 3-way transportation polytopes: Are the Graver bases for 3-way transportation polytopes as nice as they are for 2-way transportation polytopes? Do 3-way transportation polytopes have the "interval property" for entry values?

Most of these questions had easy solutions for classical transportation polytopes. In the next sections we answer all these questions for multi-way transportation polytopes.

3.2.1. Feasibility and dimension revisited. Recall Lemma 2.2 for 2-way transportation polytopes, which gave a simple characterization for a non-empty polytope in terms of its margins. From the equations in (3.1), a similar necessary and sufficient condition for the 3 -way axial transportation polytope to be non-empty can be proved:

Lemma 3.6. Let $P$ be the $p \times q \times s$ axial 3-way transportation polytope defined by the marginals $u, v$, and $w$. The polytope $P$ is non-empty if and only if

$$
\sum_{i=1}^{p} u_{i}=\sum_{j=1}^{q} v_{j}=\sum_{k=1}^{s} w_{k}
$$

The proof of this lemma is like Lemma 2.2 for 2-way transportation polytopes, but using a 3-way analogue of the northwest corner rule algorithm.

While similar statements are true for $d$-way transportation polytopes defined by 1-marginals, the real feasibility problem does not have a known characterization for $m$-marginals with $m \geq 2$, even for 3 -way transportation polytopes. This study is called real feasibility or the Vlach Problems (see [141). The conditions on the 
margins proposed by Schell, Haley, Moravek and Vlack (see [141) are necessary, but not sufficient to guarantee that the polytopes are non-empty. By the universality theorem one cannot expect a simple characterization (in terms of the 2-marginals of 3-way transportation polytope) to decide when they are empty. In fact, due to Theorem 3.3. given a prescribed collection of marginals that seem to describe a $d$-way transportation polytope of size $p_{1} \times \cdots \times p_{d}$, deciding whether there is an integer table with these margins is an NP-complete problem.

Recall that we had also a nice simple dimension formula for 2-way transportation polytopes. As a consequence of Lemma 3.6 the 3-way axial transportation polytope $P$ defined by (3.1) is completely described by only $p+q+s-2$ independent equations. The maximum possible dimension for $p \times q \times s$ transportation polytopes defined by 1-marginals is $p q s-p-q-s+2$. For planar 3-way transportation polytopes, one can see that in fact only $p q+p s+q s-p-q-s+1$ of the defining equations are linearly independent for feasible systems. The maximum possible dimension for $p \times q \times s$ transportation polytopes defined by 2-marginals is $(p-1)(q-1)(s-1)$. Unfortunately, by the universality theorem, the dimension of these polytopes can be any number up to $(p-1)(q-1)(s-1)$.

3.2.2. Combinatorics of faces revisited. From the universality theorem one can expect that the $f$-vector and indeed the entire combinatorial properties of any rational polytope will appear when listing all the $f$-vectors of 3-way transportation polytopes. Indeed, Shmuel Onn has suggested this as a way to systematically enumerate combinatorial types of polytopes. In [58], there was an experimental investigation of the possible polyhedra that arise for small 3-way transportation polytopes. The number of vertices of certain low-dimensional 3-way transportation polytopes have been completely classified:

Theorem 3.7. The possible numbers of vertices of non-degenerate $2 \times 2 \times 2$ and $2 \times 2 \times 3$ axial transportation polytopes are those given in Table 3 .

Moreover, every non-degenerate $2 \times 2 \times 4$ axial transportation polytope has between 32 and 504 vertices. Every non-degenerate $2 \times 3 \times 3$ axial transportation polytopes has between 81 and 1056 vertices. The number of vertices of non-degenerate $3 \times 3 \times 3$ axial transportation polytopes is at least 729 .

Theorem 3.8. The possible numbers of vertices of non-degenerate $2 \times 2 \times 2,2 \times 2 \times 3$, $2 \times 2 \times 4,2 \times 2 \times 5$, and $2 \times 3 \times 3$ planar transportation polytopes are those given in Table 4. Moreover, every non-degenerate $2 \times 3 \times 4$ planar transportation polytope has between 7 and 480 vertices.

\begin{tabular}{|c|c|c|}
\hline Size & Dimension & Possible numbers of vertices \\
\hline $2 \times 2 \times 2$ & 4 & 811 14 \\
\hline $2 \times 2 \times 3$ & 7 & 1824303236384042444648505254565860626466 \\
& & 68707274767880848696108 \\
\hline
\end{tabular}

TABLE 3. Numbers of vertices possible in non-degenerate axial transportation polytopes

Again from Theorem 3.3 , the graph of every rational convex polyhedron will appear as the graph of some 3-way transportation polytope. In particular if the Hirsch Conjecture is true for 3 -way transportation polytopes given by 2-marginals, 


\begin{tabular}{|c|c|c|}
\hline Size & Dimension & Possible numbers of vertices \\
\hline $2 \times 2 \times 2$ & 1 & 2 \\
\hline $2 \times 2 \times 3$ & 2 & 3456 \\
\hline $2 \times 2 \times 4$ & 3 & 4681012 \\
\hline $2 \times 2 \times 5$ & 4 & 5811121415161718192021222324252627282930 \\
\hline $2 \times 3 \times 3$ & 4 & 58911121314151617181920212223 \\
& & 2425262728293031323334353637383940 \\
& & 41424344454647484950515253545556575859 \\
\hline
\end{tabular}

TABLE 4. Numbers of vertices possible in non-degenerate planar transportation polytopes

then it is true for all rational convex polytopes. By Corollary 3.5 the graph of every rational convex polytope is the graph of a face of some 3-way transportation polytope given by 1-margins. Interestingly, in joint work with Onn and Santos (see [58]), we proved a quadratic bound on the diameter of 3-way transportation polytopes given by 2-margins:

Theorem 3.9. The diameter of every $p \times q \times s$ axial 3-way transportation polytope is at most $2(p+q+s-2)^{2}$.

Open Problem 3.10. Prove or disprove the Linear Hirsch Conjecture for 3-way axial transportation polytopes: is it true that there is a universal constant $k$ such that the diameter of every $p \times q \times s$ axial 3-way transportation polytope is at most $k(p+q+s)$ ?

3.2.3. Integer points revisited. Integer feasibility becomes now a truly difficult problem because due to Theorem 3.3. all linear and integer programming problems are slim 3-way transportation problems. In other words, any linear or integer programming problem is equivalent to one that has a $\{0,1\}$-valued constraint matrix, with exactly three 1's per column in the constraint matrix, and depends only on the right-hand side data.

Again, there is bad news for the integer range property. Unlike Lemma 2.31 for 2 -way transportation polytopes, now the values of a variable $x_{i, j, k}$ in a 3 -way transportation polytope can have integer gaps. Similarly we saw that 2-way transportation polytopes have a nice Graver basis, which we recall is a minimal set of vectors needed to travel between any pair of integer points in the polytope. Unlike the case of 2-way transportation problems and as a consequence of Theorem 3.3 . the coefficients in the entries of a Markov basis for $d$-way transportation polytopes can be arbitrarily large (see [63]), not just $0,-1,1$ as we saw in Lemma 2.32 .

Since any integer linear programming problem can be encoded as a slim 3-way transportation problem, the family of 3 -way transportation polytopes really varied. The very same family of 3-way transportation problems of $p \times q \times 3$ and specified by 2-margins contains subproblems that admit fully polynomial approximation schemes as well as subproblems that do not have arbitrarily close approximation (unless $N P=P$ ). For this reason, no purely combinatorial approximation algorithm, i.e., one that does not take into account the 2-margin values, can be devised.

As we had for 2-way transportation polytopes, we have 3-way Birkhoff polytopes, which are much more complicated: 
Definition 3.11. The Birkhoff polytope has the following generalizations in the 3-way setting, these are the multi-way assignment polytopes:

(1) The generalized Birkhoff 3-way axial polytope is the axial 3-way transportation $p \times q \times s$ polytope whose 1-marginals are given by the vectors $u=(q s, \ldots, q s) \in \mathbb{R}^{p}, v=(p s, \ldots, p s) \in \mathbb{R}^{q}$, and $w=(p q, \ldots, p q) \in \mathbb{R}^{s}$.

(2) The generalized Birkhoff 3-way planar polytope is the planar 3-way transportation $p \times q \times s$ polytope whose 2-marginals are given by the $q \times s$ matrix $U_{j, k}=p$, the $p \times s$ matrix $V_{i, k}=q$, and the $p \times q$ matrix $W_{i, j}=s$.

There is a vibrant study of $d$-way assignment polytopes. We refer the readers to the recent book [33] about assignment problems. We would simply like to mention some results that show how much harder it is to work with them versus the 2-way Birkhoff polytope. First, a now-classical result of Karp about the axial assignment polytope shows it is much more difficult to optimize over $d$-way assignment polytopes.

Theorem 3.12 (Karp [101]). The optimization problem

$$
\begin{aligned}
\text { maximize/minimize } & \sum c_{i, j, k} x_{i, j, k} \\
\text { subject to } & \left\{\begin{array}{l}
\sum_{j, k}^{j} x_{i, j, k}=1, \\
\sum_{i, k} x_{i, j, k}=1, \\
\sum_{i, j} x_{i, j, k}=1, \\
x \in \mathbb{Z}_{\geq 0}^{p \times p \times p}
\end{array}\right.
\end{aligned}
$$

is NP-hard.

Theorem 3.13 (Crama, Spieksma [48). For the minimization problem above, no polynomial time algorithm can even achieve a constant performance ratio unless $N P=P$.

There are very interesting "universality" results about the coordinates of vertices of the generalized assignment problem with 1-margins.

Definition 3.14. For a vertex $x$ of the $d$-way 1-margin assignment problem define its spectrum to be the vector spectrum $(x)$ with positive and decreasing entries which contains the values of all entries with repetitions deleted.

For example, the spectrum of a permutation matrix is always 1 .

Gromova (see [84]) gave a characterization of which vectors are in the spectrum. Here are some of her results:

Theorem 3.15 (Gromova 84]). Given a positive decreasing vector $\sigma$ of rational numbers its relation matrix $R(\sigma)$ is the matrix of all distinct non-negative integer row vectors $\tau$ such that $\sigma \cdot \tau=1$

(1) For any positive decreasing vector $v$ with components less than one whose relation matrix is not empty and whose columns are linearly independent and for $k \geq \max \left(1 / v_{i}\right)$, there is a vertex of a $d$-way assignment polytope with 1-margins with spectrum $v$. 
(2) Take any positive decreasing vector $v$ with components less than one. It appears as part of the spectrum of some vertex of a 3-way assignment polytope.

We now discuss $d$-way assignment polytopes defined by 2-margins. Recall the Birkhoff-von Neumann Theorem (Theorem 2.12 which stated that the $p \times p$ Birkhoff polytope has $p$ ! vertices. There is a 3-way analogue of this result: First, recall the $p \times p \times p$ generalized Birkhoff planar polytope (also called the 2-marginals assignment polytope) is the 3-way transportation polytope of line sums whose 2margins are given by $U_{j, k}=V_{i, k}=W_{i, j}=1$. In the case of 2-margins one can see that a solution, which is a 3 -way array, has in each planar slice a permutation matrix. This indicates that there is a bijection between the $0 / 1$ vertices of the 3 way 2-marginals $p \times p \times p$ assignment polytope and the possible $p \times p$ latin squares. Although their number is not known exactly this is enough to say that the number of $0 / 1$ vertices of the polytope is bounded below by $(p !)^{2 p} / p^{p^{2}}$. Recently, Linial and Luria (see [109]) proved that the total number of vertices of the $p \times p \times p$ generalized Birkhoff polytope is at least exponential in the number of Latin squares of order $p$.

Again we have a hardness result on the planar 3-way assignment polytope:

Theorem 3.16. (Dyer, Frieze [70) The linear optimization problem

$$
\begin{array}{ll}
\text { maximize } & \sum c_{i, j, k} x_{i, j, k} \\
\text { subject to } & \left\{\begin{array}{l}
\sum_{i} x_{i, j, k}=1, \\
\sum_{j} x_{i, j, k}=1, \\
\sum_{k}^{j} x_{i, j, k}=1, \\
x \in \mathbb{Z}_{\geq 0}^{p \times p \times p}
\end{array}\right.
\end{array}
$$

is in general NP-hard, even when $c_{i, j, k} \in\{0,1\}$. However, when $c_{i, j, k}=c_{i, j, l}$ for all $l, k$ then the problem is polynomially solvable.

Though this maximization problem is NP-hard, we note that Nishizeki and Chiba (see [119]) showed that a PTAS exists.

\section{FURTHER RESEARCH DIRECTIONS AND MORE OPEN PROBLEMS}

There are several fascinating areas of research where tables with prescribed sums of their entries play a role. In this last section we would like to take a quick look at some of these areas and highlight some very nice open questions.

4.1. 0-1 tables. We have seen some results like Birkhoff's theorem on permutation matrices that deal specifically with 0-1 tables. Interesting problems about 0-1 tables appear naturally in combinatorial representation theory (see [5, 124]) and number theory (see [3]). Analyzing the properties of $0-1$ tables is a classic area of research in combinatorial matrix theory. This field combines techniques from combinatorics and group theory and it is so large we do not even attempt to summarize the results available. The reader should consult the books [24, 30. As a taste of the richness of the field of combinatorial matrix theory let us just talk about results known for subpolytopes of the Birkhoff polytope. One can consider permutation polytopes, obtained as the convex hull of some vertices of a Birkhoff polytope. (Note that 
this notion of permutation polytope is distinct from the permutation polytopes of Billera and Sarangarajan in [16].) In [120], Onn analyzed the geometry, complexity and combinatorics of permutation polytopes. In [13, Baumeister et al. studied the faces and combinatorial types that appear in small permutation polytopes. Brualdi (see [23]) investigated the faces of the convex polytope of doubly stochastic matrices which are invariant under a fixed row and column permutation. The pth tridiagonal Birkhoff polytope is the convex hull of the vertices of the Birkhoff polytope whose support entries are in $\{(i, j) \in[p] \times[p]|| i-j \mid \leq 1\}$. In [51, da Fonseca et al. counted the number of vertices of tridiagonal Birkhoff polytopes. In [45, Costa et al. presented a formula counting the number of faces tridiagonal Birkhoff polytopes. Volumes of permutation polytopes were studied in 32 .

Costa et al. (see [43]) defined a pth acyclic Birkhoff polytope to be any polytope that is the convex hull of the set of matrices whose support corresponds to (some subset of) a fixed tree graph's edges (including loops). In [44, Costa et al. counted the faces of acyclic Birkhoff polytopes. In 43], Costa et al. proved an upper bound on the diameter of acyclic Birkhoff polytopes, which generalized the diameter result of Dahl in 52 .

Let the pth even Birkhoff polytope be the convex hull of the $\frac{1}{2} p$ ! permutation matrices corresponding to even permutations. In [50, Cunningham and Wang confirmed a conjecture of Brualdi and Liu (see [29]) that the $p$ th even Birkhoff polytope cannot be described as the solution set of polynomially many linear inequalities. In 96, Hood and Perkinson described some of the facets of the even Birkhoff polytope and proved a conjecture of Brualdi and Liu (see [29]) that the number of facets of the $p$ th even Birkhoff polytope is not polynomial in $p$. In [142, von Below showed that the condition of Mirsky given in [114] is not sufficient for determining membership of a point in an even Birkhoff polytope. Cunningham and Wang (see 50]) also investigated the membership problem for the even Birkhoff polytope. In [143], von Below and Rénier described even and odd diagonals in even Birkhoff polytopes. In [41, Cho and Nam introduced a signed analogue of the Birkhoff polytope.

The 0-1 points of transportation polytopes also have a strong connection to discrete tomography, which considers the problem of reconstructing binary images (or finite subsets of objects placed in a lattice) from a small number of their projections. The connection to tables is clear as one can think of the position of the objects in points in a grid as the placement of 0's and 1's in entries of a table. This is a very active field of research. See [4, 31, 75, 76, 77, 78, 79, 80, 81, 82, 90, 91] and the references therein. These reconstruction problems are important in CAT scanner development, electron microscope image reconstruction, and quality control in semiconductor production (see, e.g., [4, 75, 76] and the references therein).

In light of this discussion, the following open problem is interesting:

Open Problem 4.1. What is the complexity of counting all 2-way 0-1 tables for given margins?

In the next section, we discuss what is known about enumerating contingency tables in general.

4.2. Enumeration, sampling and optimization. We have seen that counting contingency tables is quite important in combinatorics and statistics. In [61, De Loera and Onn gave a complete description of the computational complexity of existence, counting, and entry-security in multi-way table problems. The following 
theorem summarizes what is known about counting (specified in terms of binary encoding or unary encoding of data):

Theorem 4.2. The computational complexity of the counting problem for integral 3 -way tables of size $p \times q \times s$ with $2 \leq p \leq q \leq s$ and all 2 -marginals specified is provided by the following table:

\begin{tabular}{|c|c|c|c|c|}
\hline & $\begin{array}{c}p, q, s \\
\text { fixed }\end{array}$ & $\begin{array}{c}p, q \text { fixed, } \\
s \text { variable }\end{array}$ & $\begin{array}{c}p \text { fixed, } \\
q, s \text { variable }\end{array}$ & $p, q, s$ variable \\
\hline unary 2-marginals & $P$ & $P$ & $\# P C$ & $\# P C$ \\
binary 2-marginals & $P$ & $\# P C$ & $\# P C$ & $\# P C$ \\
\hline
\end{tabular}

Using the highly-structured Graver bases of transportation polytopes with special restrictions one can do some polynomial-time optimization on highly difficult problems: E.g., De Loera, Hemmecke, Onn, and Weismantel (see [57]) proved there is a polynomial time algorithm that, given $s$ and fixing $p$ and $q$, solves integer programming problems of 3-way transportation polytopes of size $p \times q \times s$ defined by 2-marginals, over any integer objective. Later on, in [56] De Loera, Hemmecke, Onn, Rothblum, and Weismantel presented a polynomial oracle-time algorithm to solve convex integer maximization over 3-way planar transportation polytopes, if two of the margin sizes remain fixed. More recently (in 93]) Hemmecke, Onn, and Weismantel proved a similar result for convex integer minimization.

4.3. More open problems on transportation polytopes. We will also mention some more conjectures and open problems on transportation polytopes, and where applicable, give an update on problems where there are solutions and partial answers. We hope this will help to increase the interest in this subject.

Conjecture 4.3. It is impossible to have $p \times q \times s$ non-degenerate 3-way transportation polytopes, specified by 2-margin matrices $U, V, W$, whose number $f_{0}$ of vertices satisfies the inequalities $(p-1)(q-1)(s-1)+1<f_{0}(M(U, V, W))<$ $2(p-1)(q-1)(s-1)$ ?

This conjecture is true when $p, q, s \leq 3$.

Open Problem 4.4. Is it true that the graph of any 2-way $p \times q$ transportation polytope is Hamiltonian?

Hamiltonicity of the graph is known to hold for small values of $p$ and $q$.

Open Problem 4.5. Suppose $\phi_{1}(p, q), \phi_{2}(p, q), \ldots, \phi_{t_{p, q}}(p, q)$ are all possible values of the number of vertices of $p \times q$ transportation polytopes. Give a formula for $t_{p, q}$.

This is related to the problem of enumerating all triangulations or chambers of a vector configuration.

Conjecture 4.6. All integer numbers between 1 and $p+q-1$, and only these, are realized as the diameters of $p \times q$ transportation polytopes.

Open Problem 4.7. What are the possible number of facets for 3 -way $p \times q \times s$ non-degenerate transportation polytopes given by 2 -margins?

Open Problem 4.8. What is the largest possible number of vertices in a 3-way $p \times q \times s$ transportation polytope? 
Recall the $p \times q \times s$ generalized central transportation polytope is the 3 -way transportation polytope of line sums whose 2-margins are given by the $q \times s$ matrix $U_{j, k}=p$, the $p \times s$ matrix $V_{i, k}=q$, and the $p \times q$ matrix $W_{i, j}=s$. Yemelichev, Kovalev, Kratsov stated in [146] the conjecture that the generalized central transportation polytope had the largest number of vertices among 3-way transportation polytopes. This conjecture was proved to be false in [58]. Here are explicit 2marginals for a $3 \times 3 \times 3$ transportation polytope which has more vertices $(270$ vertices) than the generalized central transportation polytope, with only 66 vertices:

\begin{tabular}{|ccc||ccc||ccc|}
\hline 164424 & 324745 & 127239 & 163445 & 49395 & 403568 & 184032 & 123585 & 269245 \\
\hline 262784 & 601074 & 9369116 & 1151824 & 767866 & 8313284 & 886393 & 6722333 & 935582 \\
\hline 149654 & 7618489 & 1736281 & 1609500 & 6331023 & 1563901 & 1854344 & 302366 & 9075926 \\
\hline
\end{tabular}

\section{ACKNOWLEGEMENTS}

We thank Raymond Hemmecke, Fu Liu, Shmuel Onn, Francisco Santos, and Ruriko Yoshida for their suggestions and joint work on transportation problems. We are also grateful to them and Andreas Alpers, Matthias Beck, Steffen Borgwardt, Persi Diaconis, Peter Gritzmann, Igor Pak, Seth Sullivant, and Ernesto Vallejo for suggestions. The first author is grateful for the support received from NSF grant DMS-0914107. The first author is grateful to the Technische Universität München for the hospitality received during the days while writing this survey.

\section{REFERENCES}

[1] M. M. Ahmed. Algebraic Combinatorics of Magic Squares. PhD thesis, University of California, Davis, 2004.

[2] M. M. Ahmed. Polytopes of magic labelings of graphs and the faces of the Birkhoff polytope. Annals of Combinatorics, 12:241-269, 2008.

[3] A. Alpers and R. Tijdeman. The two-dimensional Prouhet-Tarry-Escott problem. J. Number Theory, 123(2):403-412, 2007.

[4] A. Alpers, P. Gritzmann. On stability, error correction, and noise compensation in discrete tomography. SIAM J. Disc. Math., 20(1):227-239, 2006.

[5] D. Avella-Alaminos and E. Vallejo. Kronecker products and the RSK correspondence. Discrete Math., 312(8):1476-1486, 2012.

[6] M. Baiöu, M. L. Balinski. The stable allocation (or ordinal transportation) problem. Math. Oper. Res. 27 (2002) 485-503

[7] M. L. Balinski and R. E. Gomory. A primal method for the assignment and transportation problem. Mgmt Sci., 10:578-593, 1964.

[8] M. L. Balinski. The Hirsch conjecture for dual transportation polyhedra. Math. Oper. Res., 9(4):629-633, 1984.

[9] M. L. Balinski, F. J. Rispoli. Signature classes of transportation polytopes. Math. Prog. Ser. A 60 (1993) 127-144

[10] A. Barvinok. Asymptotic estimates for the number of contingency tables, integer flows, and volumes of transportation polytopes. Int. Math. Res. Not. IMRN, (2):348-385, 2009.

[11] A. I. Barvinok. Polynomial time algorithm for counting integral points in polyhedra when the dimension is fixed. Math of Oper. Res. 19:769-779, 1994.

[12] A. I. Barvinok and K. Woods. Short rational generating functions for lattice point problems. J. Amer. Math. Soc. 16:957-979, 2003.

[13] B. Baumeister, C. Haase, B. Nill, and A. Paffenholz. On permutation polytopes. Adv. Math., 222:431-452, 2009.

[14] M. Beck, M. Cohen, J. Cuomo, and P. Gribelyuk. The number of magic squares, cubes and hypercubes. Amer. Math. Monthly, 110(8):707-717, 2003. 
[15] M. Beck and D. Pixton. The Ehrhart polynomial of the Birkhoff polytope. Discrete Comput. Geom., 30(4):623-637, 2003.

[16] L. J. Billera, A. Sarangarajan. The Combinatorics of Permutation Polytopes. DIMACS Series in Discrete Mathematics and Theoretical Computer Science, vol. 24, 1996.

[17] G. Birkhoff. Tres observaciones sobre el álgebra lineal. Univ. Nac. Tucumán. Revista A, 5:147-151, 1946.

[18] E. Bolker. Transportation polytopes. J. Combin. Th. Ser. B, 13:251-262, 1972.

[19] N. Bonifas, M. Di Summa, F. Eisenbrand, N. Hähnle and M. Niemeier. On sub-determinants and the diameter of polyhedra. 28th Symposium on Computational Geometry (SoCG 2012), Chapel Hill, North Carolina, USA, 2012.

[20] S. Borgwardt. On the Diameter of Partition Polytopes and Vertex-Disjoint Cycle Cover, Mathematical Programming, Series A, 2011.

[21] F. Bourgeois and J.-C. Lassalle. An extension of the Munkres algorithm for the assignment problem to rectangular matrices. Communications of the ACM, 14(12):802-804, 1971.

[22] G. Brightwell, J. van den Heuvel, and L. Stougie. A linear bound on the diameter of the transportation polytope. Combinatorica, 26(2):133-139, 2006.

[23] R. A. Brualdi. Convex polytopes of permutation invariant doubly stochastic matrices. $J$. Combin. Theory Ser. B, 23(1):58-67, 1977.

[24] R. A. Brualdi, D. Cvetković. A Combinatorial Approach to Matrix Theory and Its Applications. CRC Press, 2008.

[25] R. A. Brualdi and P. Gibson. Convex polyhedra of doubly stochastic matrices I. J. Combin. Theory Ser. A, 22(2):194-230, 1977.

[26] R. A. Brualdi and P. Gibson. Convex polyhedra of doubly stochastic matrices II. J. Combin. Theory Ser. B, 22(2):175-198, 1977.

[27] R. A. Brualdi and P. Gibson. Convex polyhedra of doubly stochastic matrices III. J. Combin. Theory Ser. A, 22(3):338-351, 1977.

[28] R. Brualdi and P. Gibson. Convex polyhedra of doubly stochastic matrices IV. Linear Algebra Appl., 15:153-172, 1976.

[29] R. A. Brualdi and B. Liu. The polytope of even doubly stochastic matrices. J. Combin. Theory Ser. A, 57(2):243-253, 1991.

[30] R. A. Brualdi, H. J. Ryser. Combinatorial Matrix Theory. Cambridge University Press, 1991.

[31] S. Brunetti, A. Del Lungo, P. Gritzmann, S. de Vries. On the reconstruction of binary and permutation matrices under (binary) tomographic constraints. Theor. Comp. Sci. 406:63-71, 2008.

[32] K. Burgraff, J. A. De Loera, and M. Omar. On volumes of permutation polytopes, In Discrete Geometry and Optimization, editors (K. Bezdek, A. Deza, and Y. Ye), vol. 69 of Fields Institute Communications,

[33] R. Burkard, M. Dell'Amico, and S. Martello. Assignment Problems. SIAM, Philadelphia, 2009.

[34] L. Buzzigoli and A. Giusti. An algorithm to calculate the lower and upper bounds of the elements of an array given its marginals. In Statistical Data Protection: Proceedings of the Conference, pages 131-147. EUROSTAT, 1999.

[35] E. R. Canfield and B. D. McKay. The asymptotic volume of the Birkhoff polytope. Available at http://arxiv.org/abs/0705.2422 2007.

[36] L. Carlitz. Enumeration of symmetric arrays. Duke Math. J., 33:771-782, 1966.

[37] C. S. Chan and D. P. Robbins. On the volume of the polytope of doubly stochastic matrices. Experiment. Math, 8(3):291-300, 1999.

[38] Y. Chen. Conditional Inference on Tables with Structural Zeros. J. of Computational and Graphical Statistics, 16(2):445-576, 2007.

[39] Y. Chen, P. Diaconis, S. P. Holmes, J. S. Liu. Sequential Monte Carlo Methods for Statistical Analysis of Tables. J. Amer. Stat. Assoc., 100(469):109-120, 2005.

[40] Y. Chen, I. H. Dinwoodie, S. Sullivant. Sequential importance sampling for multiway tables. Ann. Stat. 34(1):523-545, 2006.

[41] S. Cho and Y. Nam. Convex polytopes of generalized doubly stochastic matrices. Comm. KMS, 16(4):679-690, 2001.

[42] S. Chowdhury, G. T. Duncan, R. Krishnan, S. F. Roehrig, and S. Mukherjee. Disclosure detection in multivariate categorical databases: Auditing confidentiality protection through two new matrix operators. Mgmt Sci., 45:1710-1723, 1999. 
[43] L. Costa, C. M. da Fonseca, and E. A. Martins. The diameter of the acyclic Birkhoff polytope. Linear Algebra Appl., 428:1524-1537, 2008.

[44] L. Costa, C. M. da Fonseca, and E. A. Martins. Face counting on an Acyclic Birkhoff polytope. Linear Algebra Appl., 430(4):1216-1235, 2009.

[45] L. Costa, C. M. da Fonseca, and E. A. Martins. The number of faces of the tridiagonal Birkhoff polytope. J. Math. Sci., 161(6):867-877, 2009.

[46] L. H. Cox. Bounds on entries in 3-dimensional contingency tables subject to given marginal totals. In Inference Control in Statistical Databases - From Theory to Practice, volume 2316 of Lecture Notes in Computer Science, pages 21-33, New York, NY, 2002. Springer-Verlag.

[47] L. H. Cox. On properties of multi-dimensional statistical tables. J. Statist. Plann. Inference, 117:251-273, 2003.

[48] Y. Crama, F. C. R. Spieksma. Approximation algorithms for three-dimensional assignment problems with triangle inequalities. Eur. J. Oper. Res. 60:273-279, 1992.

[49] M. Cryan, M. Dyer, H. Müller, L. Stougie. Random walks on the vertices of transportation polytopes with constant number of sources. Proc. 14th Ann. ACM-SIAM Symp. Disc. Alg. (Baltimore, MD) 330-339, ACM, New York, 2003

[50] W. H. Cunningham and Y. Wang. On the even permutation polytope. Linear Algebra Appl., 389:269-281, 2004.

[51] C. M. da Fonseca and E. Marques de Sá. Fibonacci numbers, alternating parity sequences and faces of the tridiagonal Birkhoff polytope. Discrete Math., 308:1308-1318, 2008.

[52] G. Dahl. Tridiagonal doubly stochastic matrices. Linear Algebra Appl., 390:197-208, 2004.

[53] G. Dahl. Transportation matrices with staircase patterns and majorization. Linear Algebra Appl., 429(7):1840-1850, 2008.

[54] J. A. De Loera. The many aspects of counting lattice points in polytopes. Mathematische Semesterberichte, 52(2):175-195, 2005.

[55] J. A. De Loera, R. Hemmecke, and M. Köppe Algebraic and geometric ideas in the theory of discrete optimization. MOS-SIAM Series on Optimization, 14. SIAM-MPS, Philadelphia, PA; 2013.

[56] J. A. De Loera, R. Hemmecke, S. Onn, U. G. Rothblum, and R. Weismantel. Convex integer maximization via Graver bases. J. Pure and Applied Algebra, 213:1569-1577, 2009.

[57] J. A. De Loera, R. Hemmecke, S. Onn, R. Weismantel. $N$-fold integer programming. Disc. Optim., 5:231-241, 2008. (Volume in memory of George B. Dantzig.)

[58] J. A. De Loera, E. D. Kim, S. Onn, and F. Santos. Graphs of transportation polytopes. J. Combin. Theory Ser. A, 116(8):1306-1325, 2009.

[59] J. A. De Loera, S. Klee. Transportation Problems and Simplicial Polytopes That Are Not Weakly Vertex-Decomposable. Math. Oper. Res., 37(4):670-674, 2012.

[60] J. A. De Loera, F. Liu, and R. Yoshida. A generating function for all semi-magic squares and the volume of the Birkhoff polytope. J. Algebraic Combin., 30(1):113-139, 2009.

[61] J. A. De Loera, S. Onn. The complexity of three-way statistical tables. SIAM J. Comp. 33 (2004) 819-836

[62] J. A. De Loera, S. Onn. All linear and integer programming problems are slim 3-way transportation programs. SIAM J. of Optimization, 17(3):806-821, 2006.

[63] J. A. De Loera, S. Onn. Markov bases of three-way tables are arbitrarily complicated. J. Symbolic Computation, 41:173-181, 2006.

[64] P. Diaconis and A. Gamburd. Random matrices, magic squares and matching polynomials. Electronic J. Combinatorics, 11(2), 2004.

[65] P. Diaconis, A. Gangolli. Rectangular arrays with fixed margins. In: Discrete Probability and Algorithms (Minneapolis, MN, 1993), IMA Vol. Math. App. 72 15-41, Springer, New York, 1995 [66] A. Dobra and S. E. Fienberg. Bounds for cell entries in contingency tables given marginal totals and decomposable graphs. In Proceedings of the National Academy of Sciences 97, pages 11185-11192, 2000.

[67] G. T. Duncan, S. E. Fienberg, R. Krishnan, R. Padman, and S. F. Roehrig. Disclosure limitation methods and information loss for tabular data. In P. Doyle, J. I. Land, J. M. Theeuwes, and L. V. Zayatz, editors, Confidentiality, Disclosure and Data Access: Theory and Practical Applications for Statistical Agencies. North-Holland, 2001.

[68] G. T. Duncan, R. Krishnan, R. Padman, and S. F. Roehrig. Disclosure detection in multiple linked categorical datafiles: A unified network approach. In Statistical Data Protection: Proceedings of the Conference, pages 149-162. EUROSTAT, 1999. 
[69] M. Dyer and A. Frieze. Random walks, totally unimodular matrices, and a randomised dual simplex algorithm. Math. Program., 64:1-16, 1994.

[70] M. Dyer and A. Frieze. Planar 3dm is NP-complete. J. of Algorithms, 7:174184, 1983.

[71] M. Dyer, D. Mount, R. Kannan, J. Mount. Sampling contingency tables. Random Structures and Algorithms, 10:487-506, 1997.

[72] J. Edmonds. Paths, trees, and flowers. Canad. J. Math., 17:449-467, 1965.

[73] J. Edmonds and R. M. Karp. Theoretical improvements in algorithmic efficiency for network flow problems. In Combinatorial Structures and their Applications (Proc. Calgary Internat. Conf., Calgary, Alta., 1969), pages 93-96. Gordon and Breach, New York, 1970.

[74] S. E. Fienberg. Fréchet and Bonferroni bounds for multi-way tables of counts with applications to disclosure limitation. In Statistical Data Protection: Proceedings of the Conference, pages 115-129. EUROSTAT, 1999.

[75] R. J. Gardner, P. Gritzmann. Discrete tomography: determination of finite sets by X-rays. Trans. Amer. Math. Soc., 349(6):2271-2295, 1997.

[76] R. J. Gardner, P. Gritzmann. Successive determination and verification of polytopes by their X-rays. J. London Math. Soc., 50(2):375-391, 1994.

[77] R. J. Gardner, P. Gritzmann, D. Prangenberg. On the computational complexity of reconstructing lattice sets from their X-rays. Discrete Math., 202:45-71, 1999.

[78] P. Gritzmann, B. Langfeld. On the index of Siegel grids and its application to the tomography of quasicrystals. Eur. J. Comb., 29:1894-1909, 2008.

[79] P. Gritzmann, B. Langfield, M. Wiegelmann. Uniqueness in discrete tomography: three remarks and a corollary. SIAM J. Discrete Math., 25(4):1589-1599, 2011.

[80] P. Gritzmann, D. Prangenberg, S. de Vries, M. Wiegelmann. Success and Failure of Certain Reconstruction and Uniqueness Algorithms in Discrete Tomography. Intl. J. Imaging Syst. Tech., 9:101-109, 1998.

[81] P. Gritzmann, S. de Vries. Approximating binary images from discrete X-rays. SIAM J. Optim., 11(2):522-546, 2000.

[82] P. Gritzmann, S. de Vries. On the algorithmic inversion of the discrete Radon transform. Theor. Comp. Sci., 281:455-469, 2002.

[83] J. E. Graver. On the foundation of linear and integer programming I. Math. Prog. 9:207226, 1975.

[84] M. B. Gromova. The Birkhoff-von Neumann theorem for polystochastic matrices. In Operations research and statistical simulation, No. 2 (Russian). 315, 149, Izdat. Leningrad. Univ., Leningrad, 1974.

[85] B. Grünbaum. Convex Polytopes. Number 221 in Graduate Texts in Mathematics. SpringerVerlag, New York, NY, 2nd edition, 2003.

[86] K. Haley. The multi-index problem. Oper. Res., 11(3):368-379, 1963.

[87] K. B. Haley. Note on the letter by Morávek and Vlach. Opns. Res., 15:545-546, 1967.

[88] E. Q. Halleck. Magic squares subclasses as linear Diophantine systems. PhD thesis, University of California, San Diego, 2000.

[89] D. J. Hartfiel. Full patterns in truncated transportation polytopes. Linear and Multilinear Algebra, 30(3):213-221, 1991.

[90] G. T. Herman and A. Kuba, editors. Discrete tomography. Applied and Numerical Harmonic Analysis. Birkhäuser Boston Inc., Boston, MA, 1999. Foundations, algorithms, and applications.

[91] G. T. Herman and A. Kuba, editors. Advances in discrete tomography and its applications. Applied and Numerical Harmonic Analysis. Birkhäuser Boston Inc., Boston, MA, 2007.

[92] R. Hemmecke. On the computation of Hilbert bases of cones. In A. M. Cohen, X. S. Gao, and N. Takayama, editors, Proceedings of First International Congress of Mathematical Software, pages 307-317. World Scientific, 2002.

[93] R. Hemmecke, S. Onn, and R. Weismantel. A polynomial oracle-time algorithm for convex integer minimization. Math. Program. Ser. A, 2009.

[94] F. L. Hitchcock. The distribution of a product from several sources to numerous localities. $J$. Math. and Phys., 20:224-230, 1941.

[95] A. Hoffman. What the transportation problem did for me. Ann. of Oper. Res., 149(1):117$120,2007$.

[96] J. Hood and D. Perkinson. Some facets of the polytope of even permutation matrices. Linear Algebra Appl., 381:237-244, 2004.

[97] C. Hurkens. Personal communication. 2007. 
[98] R. Irving, M. R. Jerrum. Three-dimensional statistical data security problems. SIAM J. Comp. 23 (1994) 170-184

[99] W. Junginger. Mehrdimensionale Transportprobleme, Habilitationsschrift, Universität Stuttgart, Stuttgart, 1976.

[100] L. Kantorovich. On the translocation of masses. C.R. (Doklady) Acad. Sci. URSS (N.S.), 37:199-201, 1942

[101] R. M. Karp. Reducibility Among Combinatorial Problems. Complexity of Computer Computations 85-103, 1972.

[102] E. Klafszky and T. Terlaky. Variants of the Hungarian method for solving linear programming problems. Optimization, 20(1):79-91, 1989.

[103] V. Klee, C. Witzgall. Facets and vertices of transportation polytopes. In: Mathematics of the Decision Sciences, Part I (Stanford, CA, 1967), 257-282, AMS, Providence, RI, 1968

[104] D. Kőnig. Gráfok és alkalmazásuk a determinánsok és a halmazok elméletére. Matematikai és Természettudományi Értesítő, 34:104-119, 1916.

[105] D. Kőnig. Über Graphen und ihre Andwendung auf Determinantentheorie und Mengelehre. Math. Ann., 77:453-465, 1916.

[106] T. C. Koopmans. Optimum Utilization of the Transportation System. In Proc. International Statistical Conferences 5:136-146, 1947.

[107] H. W. Kuhn. The Hungarian method for the assignment problem. Naval Res. Logis. Quarterly, 2:83-97, 1955.

[108] H. W. Kuhn. Variants of the Hungarian method for assignment problems. Naval Res. Logis. Quarterly, 3:253-258, 1956.

[109] N. Linial, Z. Luria. On the vertices of the $d$-dimensional Birkhoff polytope. http://arxiv . org/pdf/1208.4218v1.pdf

[110] F. Liu. Perturbation of transportation polytopes Journal of Combinatorial Theory Ser. A 120 (2013), no. 7, 1539-1561.

[111] L. Lovász and M. D. Plummer. Matching Theory. North-Holland, Amsterdam, 1986.

[112] J. Matoušek and B. Gärtner. Understanding and Using Linear Programming. Universitext. Springer-Verlag, Berlin, 2007.

[113] C. R. Mehta and N. R. Patel. A network algorithm for performing Fisher's exact test in $r \times c$ contingency tables. J. Amer. Statist. Assoc., 78:427-434, 1983.

[114] L. Mirsky. Even doubly stochastic matrices. Math. Ann., 144:418-421, 1961.

[115] J. Morávek and M. Vlach. On the necessary conditions for the existence of the solution of the multi-index transportation problem. Oper. Res., 15(3):471-474, 1967.

[116] J. Morávek and M. Vlach. On necessary conditions for a class of systems of linear inequalities. Aplikace Matematiky, 13:299-303, 1968.

[117] T. S. Motzkin. The multi-index transportation problem. Bull. Amer. Math. Soc., 58:494, 1952.

[118] J. Munkres. Algorithms for the assignment and transportation problems. J. Soc. Indust. Appl. Math., 5(1):32-38, 1957.

[119] T. Nishizeki, N. Chiba. Planar graphs: Theory and algorithms. In Volume 32 of Annals of Discrete Mathematics. Elsevier, Amsterdam. 1988.

[120] S. Onn. Geometry, complexity, and combinatorics of permutation polytopes. J. Combin. Theory Ser. A, 64:31-49, 1993.

[121] S. Onn. Nonlinear Discrete Optimization. Zurich Lectures in Advanced Mathematics, European Mathematical Society, 2010

[122] I. Pak. Four questions on Birkhoff Polytope. Annals of Cominatorics 4:83-90, 2000.

[123] I. Pak. On the number of faces of certain transportation polytopes. European J. of Comb., 21(5):689-694, 2000.

[124] I. Pak. Hook length formula and geometric combinatorics. Sém. Lothar. Combin., 46:Art. B46f, 13 pp. (electronic), 2001/02.

[125] D. Pixton. The Volumes of Birkhoff polytopes for $n \leq 10$. Available at http://www.math. binghamton.edu/dennis/Birkhoff/volumes.html

[126] J. S. Provan and L.J. Billera. Decompositions of simplicial complexes related to diameters of convex polyhedra. Math. Oper. Res. 5(4):576-594, 1980.

[127] M. Queyranne and F. C. R. Spieksma. Multi-index transportation problems. In C. A. Floudas and P. Pardalos, editors, Encyclopedia of Optimization, volume III, pages 450-456. Kluwer, 2001.

[128] F. Santos. A counterexample to the Hirsch Conjecture. Ann. Math., 176(1):383-412, 2012. 
[129] E. Schell. Distribution of a product by several properties. In Proceedings of the 2nd symposium on Linear Programming, volume 2, Washington, 1955.

[130] A. Schrijver. Theory of Linear and Integer Programming. Wiley-Interscience Series in Discrete Mathematics and Optimization. Wiley, Chichester, England, 1998.

[131] G. Smith. Further necessary conditions for the existence of a solution to the multi-index problem. Oper. Res., 11(3):380-386, 1973.

[132] G. Smith. A procedure for determining necessary and sufficient conditions for the existence of a solution to the multi-index problem. Aplikace Matematiky, 19:177-183, 1974.

[133] G. Smith. On the Morávek and Vlach conditions for the existence of a solution to the multi-index problem. Applications of Mathematics, 20(6):432-435, 1975.

[134] R. P. Stanley. Linear homogeneous Diophantine equations and magic labelings of graphs. Duke Math. J., 40:607-632, 1973.

[135] R. P. Stanley. Magic labelings of graphs, symmetric magic squares, systems of parameters, and Cohen-Macaulay rings. Duke Math. J., 43(3):511-531, 1976.

[136] E. Steinitz. Über die Konstruction der Configurationen $n$ (sub 3). PhD thesis, Universität Breslau, 1894

[137] L. Stougie. A polynomial bound on the diameter of the transportation polytope, 2002.

[138] N. Tomizawa. On some techniques useful for solution of transportation network problems. Networks, 1:173-194, 1971.

[139] Transportation Polytope Database. Available at http://www.math.ucdavis.edu/ deloera/ TRANSPORT_DATABASE/

[140] J. van den Heuvel and L. Stougie. A quadratic bound on the diameter of the transportation polytope.

[141] M. Vlach. Conditions for the existence of solutions of the three-dimensional planar transportation problem. Disc. App. Math. 13 (1986) 61-78

[142] J. von Below. On a theorem of L. Mirsky on even doubly-stochastic matrices. Discrete Math., 55(3):311-312, 1985.

[143] J. von Below and S. Rénier. Even and odd diagonals in doubly stochastic matrices. Discrete Math., 308(17):3917-3929, 2008.

[144] J. von Neumann. A certain zero-sum two-person game equivalent to an optimal assignment problem. Ann. Math. Studies, 28:5-12, 1953.

[145] J. Xi, R. Yoshida, D. Haws. Estimating the number of zero-one multi-way tables via sequential importance sampling

[146] V. A. Yemelichev, M. M. Kovalev, M. K. Kravtsov. Polytopes, Graphs and Optimisation. Cambridge Univ. Press, Cambridge, 1984

[147] G. M. Ziegler. Lectures on Polytopes. Number 152 in Graduate Texts in Mathematics. Springer-Verlag, New York, NY, 1994. 